Appeared in Fundamenta Informaticae 183(1-2) : 6796(2021).

Available at IOS Press through:

https://doi.org/10.3233/FI-2021-2082

\title{
Investigating Reversibility of Steps in Petri Nets
}

\author{
David de Frutos Escrig \\ Facultad de Ciencias Matemáticas \\ Universidad Complutense de Madrid \\ 28040 Madrid, Spain \\ defrutos@sip.ucm.es
}

\section{Lukasz Mikulski*}

Faculty of Mathematics and Computer Science

Nicolaus Copernicus University

Chopina 12/18, 87-100 Toruń, Poland

lukasz.mikulski@mat.umk.pl

\author{
Maciej Koutny \\ School of Computing \\ Newcastle University \\ Newcastle upon Tyne NE1 7RU, U.K. \\ maciej.koutny@ncl.ac.uk
}

\begin{abstract}
In reversible computations one is interested in the development of mechanisms allowing to undo the effects of executed actions. The past research has been concerned mainly with reversing single actions. In this paper, we consider the problem of reversing the effect of the execution of groups of actions (steps).

Using Petri nets as a system model, we introduce concepts related to this new scenario, generalising notions used in the single action case. We then present properties arising when reverse actions are allowed in place/transition nets (PT-nets). We obtain both positive and negative results, showing that allowing steps makes reversibility more problematic than in the interleaving/sequential case. In particular, we demonstrate that there is a crucial difference between reversing steps which are sets and those which are true multisets. Moreover, in contrast to sequential semantics, splitting reverses does not lead to a general method for reversing bounded PT-nets. We then show that a suitable solution can be obtained by combining split reverses with weighted read arcs.
\end{abstract}

Keywords: Petri net, reversible computation, step semantics, action splitting, net synthesis, direct reversibility, mixed reversibility, weighted activator arcs

\footnotetext{
*Address for correspondence: Faculty of Mathematics and Computer Science, Nicolaus Copernicus University, Chopina 12/18, 87-100 Toruń, Poland.

Also affiliated at: Institute of Computer Science, Polish Academy of Sciences, Jana Kazimierza 5, 01-248 Warsaw, Poland
} 


\section{Introduction}

Reversibility of (partial) computations has been extensively studied during the past years, looking for mechanisms that allow to (partially) undo some actions executed during a computational process, that for some reason one needs to cancel. As a result, the execution can then continue from a consistent state as if that suppressed action had not been executed at all. In particular, these mechanisms allow for the correct implementation of transactions [1, 2], that are partial computations which either are totally executed or not executed at all. This includes updating in databases, so that one never commits an 'incomplete' set of related updates that might produce an inconsistent state (in which one could infer contradictory facts). Another example would be money transfers between banks, or modern e-commerce platforms, where the payments received should match the goods distributed [3].

Within Formal Methods, reversibility has been investigated, for instance, in the framework of process calculi [4, 5], event structures [6], DNA-computing [7], category theory [8], and quantum computing [9]. In the latter case, it plays a central role due to the inherent reversibility of the mechanisms on which quantum computing is based. This paper is concerned with reversibility in place/transition nets (PT-nets), which are a fundamental class of Petri nets, operating according to the step semantics in which multisets of actions (steps) are executed simultaneously.

In Petri nets, reversibility is usually understood as a global property resembling cyclicity. It was also considered in a manner closer to its process calculi meaning using symmetric nets [10] (symmetric nets have later been used to study structural symmetries of state spaces [11]). Locally defined reversibility has not yet been extensively studied within the Petri net framework. This is rather surprising as the formalisation of an action by means of a pair of pre-places and post-places provides an immediate way of defining the reverse of the actions simply by interchanging these two sets of places. There are, however, some more recent works in which reversibility is understood as cyclicity (i.e., an ability to return to the initial state from any reachable state). They are usually based on the structure theory of Petri nets [12], or an algebraic study by means of invariants [13].

From the operational point of view, one can distinguish three essential ways of reversing computational processes: backtracking, causal reversibility, and out of causal reversibility. For concurrent systems, the backtracking mode was considered, for example, in [1], where the RCCS process algebra is introduced. An investigation of causal reversibility in the Petri net context can be found, for example, in [14], where it was implemented using occurrence nets. All three ways of reversing computations were studied in [15], where biologically motivated reversing Petri nets were introduced. In all these works, one needs to enrich the original model by additional annotations or constructs. It is the memory of monitored processes for RCCS, the computation stack encoded through colours for folded occurrence nets, and atoms and bonds together with the history function for reversing Petri nets. In our approach, we are interested in studying the possibility of reversing computations in step semantics emphasizing reversing the effects, and avoiding the reachability of new states. The latter ensures that one can reach only states that are reachable by forward computations, which differentiates our approach from the out of causal reversibility discussed in [15]. We also do not equip our nets with additional external monitors which help to ensure causality. As a result, it may happen that reverses of actions that were not yet executed become enabled. This inconvenience can, however, be easily removed by suitably augmenting a PT-net being reversed to yield another net, as described in [16]. 
The approach presented in this paper is closer to inverse nets presented in [17], and so more operational. It extends the study of reversing (sequential) transition systems initiated in [16], where it was shown that the apparent simplicity of this approach is far from trivial, mainly due to the difficulty of avoiding situations where an added reverse action is executed in an inconsistent manner. Further investigation of this problem can be found in [18], while [19] considers bounded PT-nets, distinguishing between the strict reverses and effect reverses of actions. The latter deliver the effect of reversing the original actions, but possibly with a change in the way action enabling is carried out. It was shown that some transition systems which can be solved by bounded nets allow the reversal of their actions by means of single reverse actions, while in other cases the reversal is only possible if splitting of reverses is allowed (i.e., each action has a set of reverses which collectively provide means of reversing the original action).

In [19] only the sequential (interleaving) semantics of nets was considered and, in fact, several of the presented examples were just (finite) linear transition systems, taking advantage of the results presented in [20, 21], where binary words representable by Petri net were characterised. The latter problem and its consequences for reversibility has been further investigated in [22].

About this paper We consolidate and extend the results of [23], where the study of step reversing in PT-nets and (step) transition systems was initiated. We assume that the transition systems to be synthesized include information about the multisets of actions (steps) that should be executed in parallel. Reversing of the actions should preserve this step information so that the simultaneous firing of several reverse actions should correspond to the original steps at the system represented by a PT-net.

We introduce several concepts related to this new scenario, generalising notions used in the single action case. A number of straightforward definition which worked in the sequential case are no longer adequate. When looking for their adequate generalisations, we identify two 'natural' notions of step reversibility. The former (direct reversibility) only allows steps which comprise either the original actions, or the reverse actions. The latter (mixed reversibility) allows also mixing of the original and reverse actions. It turns out that these two ways of interpreting step reversibility are fundamentally different. Crucially, the direct reversibility cannot be implemented for steps which are true multisets, and so in such cases one has to look for mixed reversibility solutions. In this way, we identified a striking difference between reversing steps which are sets and those which are true multisets (when autoconcurrency of actions in system executions is allowed). However, there is still a general positive result which basically applies whenever sequential reversing is possible and the original steps can be be satisfactorily represented.

We also adapt split reverses introduced in [19]. Unfortunately, splitting is not enough to deal with all bounded PT-nets (also adding inhibitor arcs to the PT-net model does not always help). A general solution we propose uses weighted read arcs [24] (the further development of this model is out of the scope of this paper, and is left as a topic for the future work).

The paper is organised as follows. Section 2 recalls notions and notations used throughout the paper. Moreover, some basic results concerning the step transition model are given. Section 3 introduces four different ways of defining reversibility in step transition systems, including direct step reversibility and mixed step reversibility, as well as set reversibility (where a true multiset of actions is reversed in stages) and split reversibility. Section 4 demonstrates that the direct reversibility cannot be achieved in the presence of autoconcurrency. Moreover, it characterises cases where mixed reversibil- 
ity can be replaced by (more desirable) direct reversibility or set reversibility. Section 5 provides result allowing one to deal with mixed reversibility and step reversibility in an effective way, by reducing the reversibility problem to the net synthesis problem. This approach is further continued Section 6 , where lifting of sequential reversibility to step reversibility is discussed. Section 7 proposes a general solution to the step reversibility of bounded PT-nets which relies on the weighted read arcs. Finally, Section 8 contains concluding remarks.

\section{Preliminaries}

Vectors, multisets and actions An $X$-vector over a set $X$ is a mapping $\alpha: X \rightarrow \mathbb{Z}$, where $\mathbb{Z}$ is the set of all integers. For two $X$-vectors, $\alpha$ and $\beta$, the sum $(\alpha+\beta)$, difference $(\alpha-\beta)$, and lessthan-or-equal relationship $(\alpha \leq \beta)$ are defined component-wise. The support of an $X$-vector $\alpha$ is the set $\operatorname{supp}(\alpha)=\{x \in X \mid \alpha(x) \neq 0\}$. The empty $X$-vector has the empty support and is denoted by $\varnothing_{X}$ or simply by $\varnothing$, and $-\alpha$ denotes $\varnothing_{X}-\alpha$. The union of an $X$-vector $\alpha$ and a $Y$-vector $\beta$, where $X \cap Y=\varnothing$, is the $(X \cup Y)$-vector $\alpha \sqcup \beta$ such that $\left.\alpha \sqcup \beta\right|_{X}=\alpha$ and $\left.\alpha \sqcup \beta\right|_{Y}=\beta$.

Multisets over $X$ are $X$-vectors returning non-negative integers in $\mathbb{N}$, the subsets of $X$ can be identified with multisets returning 0 or 1 , and the elements of $X$ with singleton sets. The set of all multisets over $X$ is denoted by mult $(X)$. The size of $\alpha \in \operatorname{mult}(X)$ is given by $|\alpha|=\sum_{x \in X} \alpha(x)$. For $x \in X$, we denote $x \in \alpha$ whenever $\alpha(x) \geq 1$.

In what follows, e.g., $(x x z)$ denotes a multiset $\alpha$ with the support $\{x, z\}$ satisfying $\alpha(x)=2$ and $\alpha(z)=1$. Moreover, $x^{k}$ denotes a multiset $\alpha$ with the support $\{x\}$ satisfying $\alpha(x)=k$.

Throughout the paper, $\mathcal{A}$ denotes an infinite set actions, including the reverse actions and indexed reverse actions introduced in Section 3, used in step transition systems and PT-nets to model events occurring in concurrent behaviours. To simplify the presentation, we will treat a vector or multiset $\alpha$ over $T \subseteq \mathcal{A}$ as a vector or multiset over $\mathcal{A}$, assuming that $\left.\alpha\right|_{\mathcal{A} \backslash T}=\varnothing_{\mathcal{A} \backslash T}$.

Step transition systems A step transition system is a tuple $S T S=\left(S, T, \rightarrow, s_{0}\right)$ such that $S$ is a nonempty set of states, $T$ is a finite set of actions, $\rightarrow \subseteq S \times \operatorname{mult}(T) \times S$ is the set of transitions, and $s_{0} \in S$ is the initial state. The transition labels in mult $(T)$ represent simultaneous executions of groups of actions, called steps. Rather than $(s, \alpha, r) \in \rightarrow$, we can denote $s \stackrel{\alpha}{\longrightarrow} S T S$. Moreover, $s \stackrel{\alpha}{\longrightarrow}$ STS means that there is some $r$ such that $s \stackrel{\alpha}{\longrightarrow}$ STS $r$. STS is:

- a set transition system if $\alpha$ is a set, for every transition $(s, \alpha, r)$; and

- state-finite if $S$ is finite, step-finite if $\{\alpha \mid s \stackrel{\alpha}{\longrightarrow} S T S\}$ is finite, and finite if it is both stateand step-finite (and so $\rightarrow$ is finite).

In the diagrams, step transition systems are depicted as labelled directed graphs. Arcs labelled by the empty multiset are omitted.

A state $r$ is reachable from state $s$ if there are steps $\alpha_{1}, \ldots, \alpha_{k}(k \geq 0)$ and states $s_{1}, \ldots, s_{k+1}$ such that $(s=) s_{1} \stackrel{\alpha_{1}}{\longrightarrow} S T S s_{2} \ldots s_{k} \stackrel{\alpha_{k}}{\longrightarrow} S T S s_{k+1}(=r)$. We denote this by $s \stackrel{\alpha_{1} \cdots \alpha_{k}}{\longrightarrow}$ STS $r$.

The set of all states from which a state $s$ is reachable is denoted by $\operatorname{pred}_{S T S}(s), s$ is a home state if $\operatorname{pred}_{S T S}(s)=S$, and $R \subseteq S$ is a home cover of $S T S$ if $S=\bigcup_{s \in R} \operatorname{pred}_{S T S}(s)$. 
An (undirected) path from a source state $s$ to target state $r$ is a sequence $\pi=\tau_{1} \ldots \tau_{k}(k \geq 0)$, where each $\tau_{i}$ is a pair $\left(\left(s_{i}, \alpha_{i}, r_{i}\right), \zeta_{i}\right) \in(\rightarrow \times\{+,-\})$ such that either $k=0$ and $s=r$, or $k \geq 1$ and $s=\widehat{s}_{1}, \widehat{r}_{1}=\widehat{s}_{2}, \ldots, \widehat{r}_{k-1}=\widehat{s}_{k}, \widehat{r}_{k}=r$, assuming that $\widehat{s}_{i}=s_{i}$ and $\widehat{r}_{i}=r_{i}$ if $\zeta_{i}=+$, and otherwise $\widehat{s}_{i}=r_{i}$ and $\widehat{r}_{i}=s_{i}$, for every $1 \leq i \leq k$. We denote this by $\pi \in \operatorname{paths}_{S T S}(s, r)$. The signature of $\pi$ is the $\mathcal{A}$-vector $\operatorname{sign}(\pi)=\varnothing_{\mathcal{A}} \zeta_{1} \alpha_{1} \ldots \zeta_{k} \alpha_{k}$, where the $\zeta_{i}$ 's are being treated as addition and subtraction operations. For example, if $\pi=\left(\left(s^{\prime}, \alpha, s\right),-\right)\left(\left(s^{\prime}, \beta, s^{\prime \prime}\right),+\right) \in \operatorname{paths}_{S T S}\left(s, s^{\prime \prime}\right)$, then $\operatorname{sign}(\pi)=\varnothing_{\mathcal{A}}-\alpha+\beta=\beta-\alpha$.

Intuitively, $\operatorname{sign}(\pi)$ records the 'net contribution (or effect)' made by each action along the path $\pi$, with $a \in \alpha_{i}$ making a 'positive' contribution if the transition $\left(s_{i}, \alpha_{i}, r_{i}\right)$ agrees with the direction of the path, and otherwise making a 'negative' contribution. Note that $r$ is reachable from $s$ iff there is $\pi \in$ paths $_{S T S}(s, r)$ with all the $\zeta_{i}$ 's being equal to + .

In this paper, step transition systems are intended to capture (step) reachability graphs of PTnets. We will now introduce a property of step transition systems which is motivated by the state equation which holds, in particular, for PT-nets. The basic idea is that the effect of executing an action is fixed, and so does not depend on the global state in which this happens (we will make this more precise later). Capturing such a constant effect is straightforward for PT-nets, but not for step transition systems. One can, however, approximate the concept of having 'the same effect' by considering as equivalent all undirected paths with the same source and target states.

Let $\bowtie_{S T S}$ be the least equivalence relation on the set of all $\mathcal{A}$-vectors such that: $(i) \operatorname{sign}(\pi) \bowtie_{S T S}$ $\operatorname{sign}\left(\pi^{\prime}\right)$, for all $s, r \in S$ and $\pi, \pi^{\prime} \in \operatorname{paths}_{S T S}(s, r)$; and (ii) $\alpha \bowtie_{S T S} \beta$ and $\alpha^{\prime} \bowtie_{S T S} \beta^{\prime}$ imply $\alpha+\alpha^{\prime} \bowtie_{S T S} \beta+\beta^{\prime}$, for all $\mathcal{A}$-vectors $\alpha, \alpha^{\prime}, \beta$, and $\beta^{\prime}$. Intuitively, $\alpha \bowtie_{S T S} \beta$ means that executing $\alpha$ has the same effect as executing $\beta$. This leads to the following property of a step transition $S T S$ :

$\boldsymbol{C E} \operatorname{sign}(\pi) \bowtie_{S T S} \operatorname{sign}\left(\pi^{\prime}\right)$ implies $r=r^{\prime}$, for all $s, r, r^{\prime} \in S, \pi \in \operatorname{paths}_{S T S}(s, r)$, and $\pi^{\prime} \in \operatorname{paths}_{S T S}\left(s, r^{\prime}\right)$.

(constant effect)

It is the case that $\alpha \bowtie_{S T S} \beta$ implies $-\alpha \bowtie_{S T S}-\beta$ since $\pi \in \operatorname{paths}_{S T S}(s, r)$ means that there is $\pi^{\prime} \in$ paths $_{S T S}(r, s)$ such that $\operatorname{sign}\left(\pi^{\prime}\right)=-\operatorname{sign}(\pi)$. Hence we also have the following 'backward' version of the 'forward' constant effect property $C E: \operatorname{sign}(\pi) \bowtie_{S T S} \operatorname{sign}\left(\pi^{\prime}\right)$ implies $s=s^{\prime}$, for all $s, s^{\prime}, r \in S, \pi \in \operatorname{paths}_{S T S}(s, r)$, and $\pi^{\prime} \in \operatorname{paths}_{S T S}\left(s^{\prime}, r\right)$.

We are now in a position to introduce a class of step transition systems used throughout the rest of this paper. A step transition system $S T S=\left(S, T, \rightarrow, s_{0}\right)$ is a constant effect step transition system (or CEST-system) if it satisfies $C E$ as well as the following three properties, for every $s \in S$ :

$\boldsymbol{R E A} \quad s_{0} \in \operatorname{pred}_{S T S}(s)$.

(reachability)

EL $\quad s \stackrel{\varnothing}{\longrightarrow} S T S$.

(empty loops)

SEQ $\quad s \stackrel{\alpha+\beta}{\longrightarrow}$ STS implies $s \stackrel{\alpha \beta}{\longrightarrow} S T S$.

(sequentialisability)

We then obtain two immediate properties of CEST-systems.

Proposition 2.1. Let $S T S$ be a CEST-system.

1. $r=r^{\prime}$ whenever $s \stackrel{\alpha}{\longrightarrow}$ STS $r$ and $s \stackrel{\alpha}{\longrightarrow}$ STS $r^{\prime}$. 
2. $s=r$ whenever $s \stackrel{\varnothing}{\longrightarrow} S T S$.

\section{Proof:}

Part (1) follows from $C E$, and part (2) follows from part (1) and $E L$.

Proposition 2.1 (1) captures the property of forward determinism (FD) which allows one to unambiguously denote $s \oplus_{S T S} \alpha$, or $s \oplus \alpha$ if $S T S$ is clear from the context, as the state $r$ satisfying $s \stackrel{\alpha}{\longrightarrow} S T S r$ whenever $s \stackrel{\alpha}{\longrightarrow} S T S$.

Being a CEST-system still does not mean that it can be generated by a PT-net. A complete characterisation can be obtained using, e.g., theory of regions [25, 26].

Proposition 2.2. Let $s$ be a state of a CEST-system STS. If $s \oplus \alpha$ is defined and $\beta+\gamma \leq \alpha$, then $s \oplus \beta, s \oplus(\beta+\gamma)$ and $(s \oplus \beta) \oplus \gamma$ are also defined, and $(s \oplus \beta) \oplus \gamma=s \oplus(\beta+\gamma)$.

\section{Proof:}

By $s \stackrel{\alpha}{\longrightarrow} S T S$ as well as $S E Q$ and $C E$, we have $s \stackrel{\beta}{\longrightarrow}_{S T S} s \oplus \beta \stackrel{\gamma}{\longrightarrow} S T S(s \oplus \beta) \oplus \gamma$ as well as $s \stackrel{\beta+\gamma}{\longrightarrow} S T S=(\beta+\gamma)$. We therefore have $\pi=((s, \beta, s \oplus \beta),+)((s \oplus \beta, \gamma,(s \oplus \beta) \oplus \gamma),+) \in$ $\operatorname{paths}_{S T S}(s,(s \oplus \beta) \oplus \gamma)$ and $\pi^{\prime}=((s, \beta+\gamma, s \oplus(\beta+\gamma)),+) \in \operatorname{paths}_{S T S}(s, s \oplus(\beta+\gamma))$. Moreover, $\operatorname{sign}(\pi)=\beta+\gamma=\operatorname{sign}\left(\pi^{\prime}\right)$. Hence, by $C E,(s \oplus \beta) \oplus \gamma=s \oplus(\beta+\gamma)$.

We use different ways of removing transitions from a step transition system $S T S=\left(S, T, \rightarrow, s_{0}\right)$ :

$$
\begin{aligned}
& S T S^{\text {seq }}=\left(S, T,\{(s, \alpha, r) \in \rightarrow \quad|\alpha| \leq 1\}, s_{0}\right) \\
& S T S^{\text {set }}=\left(S, T,\{(s, \alpha, r) \in \rightarrow \mid \operatorname{supp}(\alpha)=\alpha\}, s_{0}\right) \\
& S T S^{\text {spike }}=\left(S, T,\{(s, \alpha, r) \in \rightarrow|| \operatorname{supp}(\alpha) \mid \leq 1\}, s_{0}\right) \\
& \left.S T S\right|_{T^{\prime}}=\left(S, T^{\prime},\left\{(s, \alpha, r) \in \rightarrow \quad \mid \alpha \in \operatorname{mult}\left(T^{\prime}\right)\right\}, s_{0}\right) \quad\left(\text { for } T^{\prime} \subseteq T\right) .
\end{aligned}
$$

That is, $S T S^{s e q}$ is obtained by only retaining singleton steps and $\varnothing$-labelled steps, $S T S^{\text {set }}$ by only retaining steps which are sets, and $S T S^{\text {spike }}$ by removing all steps which use more than one action. Moreover, $S T S$ is a sequential / set / spiking step transition system if respectively $S T S=S T S^{s e q}$ / $S T S=S T S^{\text {set }} / S T S=S T S^{\text {spike } 1}$

For step transition systems satisfying $S E Q$, checking the satisfaction of the constant effect property can be done by restricting oneself to the sequential steps.

Proposition 2.3. Let $S T S$ be a step transition system satisfying $S E Q$. Then $S T S$ satisfies $C E$ if and only if $S T S^{s e q}$ satisfies $C E$.

\section{Proof:}

We first observe that from SEQ for $S T S$ it follows that, for every $\pi \in \operatorname{paths}_{S T S}(s, r)$, there is $\pi^{\prime} \in$ $\operatorname{paths}_{S T S^{s e q}}(s, r)$ such that $\operatorname{sign}\left(\pi^{\prime}\right)=\operatorname{sign}(\pi)(*)$. Hence, we also have $\bowtie_{S T S}=\bowtie_{S T S^{s e q}}(* *)$.

$(\Longrightarrow)$ Follows from (**) and $\pi \in \operatorname{paths}_{S T S}{ }^{s e q}(s, r) \subseteq \pi \in \operatorname{paths}_{S T S}(s, r)$.

$(\Longleftarrow)$ Follows from $(*)$ and $(* *)$. 
The essence of the next result is that adding reverses of some transitions labelled by the same action in a sequential step transition system preserves the constant effect property.

Proposition 2.4. Let $S T S=\left(S, T, \rightarrow, s_{0}\right)$ be a sequential step transition system satisfying $C E$ and $S T S^{\prime}=\left(S, T \cup\{\widetilde{a}\}, \rightarrow \cup \rightarrow^{\prime}, s_{0}\right)$, where $\rightarrow^{\prime} \subseteq\{(r, \widetilde{a}, s) \mid(s, a, r) \in \rightarrow\}$ for some $a \in T$ and $\widetilde{a} \notin T$. Then $S T S^{\prime}$ satisfies $C E$.

\section{Proof:}

The result clearly holds when $\rightarrow^{\prime}$ is empty. Otherwise, we have $a \bowtie_{S T S^{\prime}}-\widetilde{a}$. For every $\mathcal{A}$-vector $\alpha$, let $\widehat{\alpha}$ be the $\mathcal{A}$-vector such that $\left.\widehat{\alpha}\right|_{\mathcal{A} \backslash\{a, \widetilde{a}\}}=\left.\alpha\right|_{\mathcal{A} \backslash\{a, \widetilde{a}\}}, \widehat{\alpha}(a)=\alpha(a)-\alpha(\widetilde{a})$, and $\widehat{\alpha}(\widetilde{a})=0$.

We observe that, for all $s, r \in S$ and $\pi \in$ paths $_{S T S^{\prime}}(s, r)$, there is $\pi^{\prime} \in \operatorname{paths}_{S T S}(s, r)$ such that $\left.\operatorname{sign}\left(\pi^{\prime}\right)=\widehat{\operatorname{sign}(\pi}\right)(*)$. Hence, we also have that $\alpha \bowtie_{S T S^{\prime}} \beta$ iff $\widehat{\alpha} \bowtie_{S T S} \widehat{\beta}$, for all $\mathcal{A}$-vectors $\alpha$ and $\beta$ (**). The result then follows from $C E$ for $S T S$ together with (*) and (**).

Let $S T S=\left(S, T, \rightarrow, s_{0}\right)$ and $S T S^{\prime}=\left(S^{\prime}, T^{\prime}, \rightarrow^{\prime}, s_{0}^{\prime}\right)$ be two step transition systems such that $T \subseteq T^{\prime}$. Then $S T S$ is included in $S T S^{\prime}$ if there is a bijection $\psi: S \rightarrow S^{\prime}$ such that $\psi\left(s_{0}\right)=s_{0}^{\prime}$ and $\left\{\left(\psi(s), \alpha, \psi\left(s^{\prime}\right)\right) \mid s \stackrel{\alpha}{\longrightarrow} S T S s^{\prime}\right\} \subseteq \rightarrow$ 2 This is denoted by $S T S \triangleleft_{\psi} S T S^{\prime}$ or $S T S \triangleleft S T S^{\prime}$, and if $\psi$ is the identity on $S$, we denote $S T S \triangleleft S T S^{\prime}$. Also, $S T S$ is isomorphic with $S T S^{\prime}$ if there is $\psi$ such that $S T S \triangleleft_{\psi} S T S^{\prime}$ and $S T S^{\prime} \triangleleft_{\psi^{-1}} S T S$. This is denoted by $S T S \simeq_{\psi} S T S^{\prime}$ or $S T S \simeq S T S^{\prime}$.

PT-nets A PT-net (short for place/transition net [27]) is a tuple $N=\left(P, T, F, M_{0}\right)$, where $P$ is a finite set of places, $T \subseteq \mathcal{A}$ is a disjoint finite set of actions $F$ is the flow function $F:(P \times T) \cup$ $(T \times P) \rightarrow \mathbb{N}$ specifying the arc weights between places and actions, and $M_{0}$ is the initial marking (markings are multisets over $P$ representing global states). It is assumed that, for every $a \in T$, there is $p \in P$ such that $F(p, a)>0$.

The triple $(P, T, F)$ is an unmarked PT-net, and $\left.N\right|_{T^{\prime}}=\left(P, T^{\prime},\left.F\right|_{\left(P \times T^{\prime}\right) \cup\left(T^{\prime} \times P\right)}, M_{0}\right)$ is the subnet of $N$ induced by $T^{\prime} \subseteq T$.

In the diagrams, PT-nets are depicted as labelled directed graphs, with circles representing places and boxes to representing actions. Markings are represented by black tokens or numbers drawn inside the circles, the arc weight of 1 is omitted, and the 0-weight arcs are not drawn.

Multisets over $T$, again called steps, represent executions of groups of actions. The effect of a step $\alpha \in \operatorname{mult}(T)$ (and, in general, a $T$-vector $\alpha$ ) is the $P$-vector $\operatorname{eff}_{N}(\alpha)=\operatorname{post}_{N}(\alpha)-\operatorname{pre}_{N}(\alpha)$, where $\operatorname{pre}_{N}(\alpha)$ and $\operatorname{post}_{N}(\alpha)$ are multisets of places such that, for every $p \in P$ :

$$
\operatorname{pre}_{N}(\alpha)(p)=\sum_{a \in T} \alpha(a) \cdot F(p, a) \quad \text { and } \quad \operatorname{post}_{N}(\alpha)(p)=\sum_{a \in T} \alpha(a) \cdot F(a, p) .
$$

A step $\alpha$ is enabled at a marking $M$ if $\operatorname{pre}_{N}(\alpha) \leq M$, and the firing of such a step leads to the marking $M^{\prime}=M+\operatorname{eff}_{N}(\alpha) 4$ This is respectively denoted by $M[\alpha\rangle_{N}$ and $M[\alpha\rangle_{N} M^{\prime}$. Note

\footnotetext{
${ }^{2}$ If $S T S$ and $S T S^{\prime}$ are CEST-systems, then $\psi$ is unique due to REA and $F D$.

${ }^{3}$ We use the term 'actions' rather than 'transitions' when referring to the elements of $T$, in order to avoid confusion with the triples $(s, \alpha, r)$ used in the definition of step transition systems.

${ }^{4} M^{\prime}$ is a multiset due to $\operatorname{pre}_{N}(\alpha) \leq M$.
} 
that it is always the case that $M[\varnothing\rangle_{N} M$, and that $M[\alpha+\beta\rangle_{N}$ implies $M[\alpha\rangle_{N} M^{\prime}[\beta\rangle_{N}$, where $M^{\prime}=M+\operatorname{eff}_{N}(\alpha)$. These two facts motivated the inclusion of $E L$ and $S E Q$ in the definition of CEST-systems.

The reachable markings of $N$ are the smallest set of markings reach ${ }_{N}$ such that $M_{0} \in \operatorname{reach}_{N}$ and if $M \in \operatorname{reach}_{N}$ and $M[\alpha\rangle_{N}$, then $M+\operatorname{eff}_{N}(\alpha) \in \operatorname{reach}_{N} . N$ is bounded if the set reach ${ }_{N}$ of all the reachable markings is finite.

The overall behaviour of $N$ can be captured by its concurrent reachability graph which is the step transition system $C R G_{N}=\left(\operatorname{reach}_{N}, T,\left\{\left(M, \alpha, M^{\prime}\right) \mid M \in \operatorname{reach}_{N} \wedge M[\alpha\rangle_{N} M^{\prime}\right\}, M_{0}\right)$. In what follows, $M \stackrel{\alpha}{\longrightarrow} N M^{\prime}$ denotes $M \stackrel{\alpha}{\longrightarrow} C R G_{N} M^{\prime}$. Note that the concurrent reachability graphs of bounded PT-nets are finite.

The concept of marking equation can be explained in the following way. Suppose that a marking $M^{\prime}$ can be reached from marking $M$ by firing a sequence of steps, e.g., $M \stackrel{\alpha_{1} \cdots \alpha_{n}}{\longrightarrow} C R G_{N} M^{\prime}$. Then

$$
M^{\prime}=M+\operatorname{eff}_{N}(\alpha) \quad M=M^{\prime}-\operatorname{eff}_{N}(\alpha) \quad \operatorname{eff}_{N}(\alpha)=M^{\prime}-M,
$$

where $\alpha=\alpha_{1}+\cdots+\alpha_{n}$. This means that the effect of executing a multiset of actions $\alpha$ is constant, as it does not depend on the starting marking nor the ending marking nor any particular way in which the actions making up $\alpha$ were fired. Moreover, the effect of actions fired along any path from $M$ to $M^{\prime}$ is constant. This motivated the inclusion of $C E$ in the definition of CEST-systems.

It is straightforward to see that $C R G_{N}$ is a CEST-system. In particular, by Eq. (1), we have $\operatorname{eff}_{N}(\operatorname{sign}(\pi))=M^{\prime}-M$, for every $\pi \in$ paths $_{C R G_{N}}\left(M, M^{\prime}\right)$. Hence, in particular, $\alpha \bowtie_{C R G_{N}} \beta$ implies eff ${ }_{N}(\alpha)=\operatorname{eff}_{N}(\beta)$. As a result, $C E$ holds.

Solving step transition systems A step transition system $S T S$ is solvable if there is a PT-net $N$ such that $S T S \simeq C R G_{N}$. This is the standard definition used in several works concerned with the synthesis of Petri nets from transition systems. In this paper, we will also use a more general notion of solvability, defined for step transition systems with multiple initial states.

A step transition system with multiple initial states is a tuple $S T S=\left(S, T, \rightarrow, S_{0}\right)$ such that the first three components are as in the definition of a step transition system, and $S_{0} \subseteq S$ is a nonempty set of initial states. Moreover, for every $r \in S_{0}, S T S_{r}=\left(S_{r}, T, \rightarrow_{r}, r\right)$ is the step transition system such that $S_{r}=\left\{s \in S \mid r \in \operatorname{pred}_{S T S}(s)\right\}$ and $\rightarrow_{r}=\rightarrow \cap\left(S_{r} \times \operatorname{mult}(T) \times S_{r}\right)$. That is, $S T S_{r}$ is $S T S$ restricted to those states which are reachable from $r$.

A step transition system with multiple initial states $S T S$ is solvable if there is an unmarked PTnet $(P, T, F)$ and a mapping $\psi: S \rightarrow \operatorname{mult}(P)$ such that $S T S_{r} \simeq_{\left.\psi\right|_{S r}} C R G_{(P, T, F, \psi(r))}$, for every $r \in S_{0}$. That is, a solution in this case is an unmarked PT-net which can be 'started' in different initial markings, each such initial marking solving one of the step transition systems which make up STS.

Example 2.5. Let us consider $S T S=\left(\left\{q_{1}, \ldots, q_{6}\right\},\{a, b, c\}, \rightarrow,\left\{q_{1}, q_{2}\right\}\right)$, a step transition system with multiple initial states depicted in Figure 1 $(a)$ (for simplicity, all nonempty steps are singletons).

The step transition system $S T S_{q_{2}}$, depicted on Figure $1(b)$, is obtained from $S T S$ by removing all the states which are not reachable from $q_{2} . S T S_{q_{1}}$ is constructed in similar way. The PT-net 
$N=\left(P, T, F,\left(p_{1} p_{4}\right)\right)$ solving $S T S_{q_{1}}$ is depicted on Figure $1(c)$. As $N=\left(P, T, F, p_{2}^{4}+p_{4}\right)$ is a solution for $S T S_{q_{2}}$, it follows that $S T S$ is solvable.
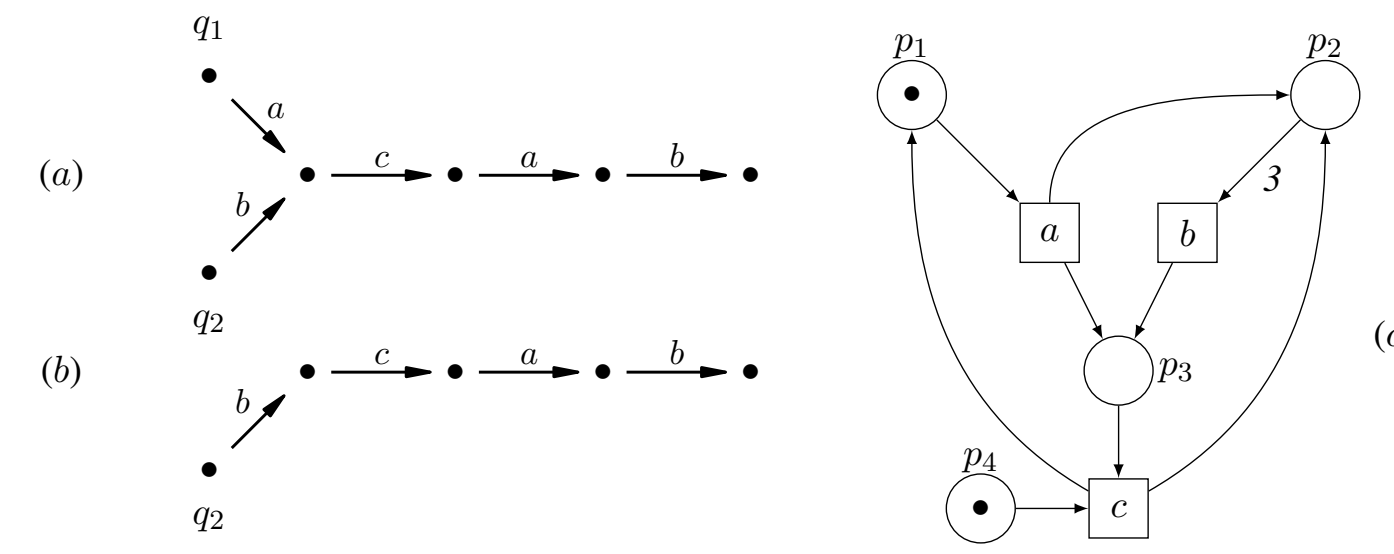

(c)

Figure 1. A step transition system with multiple initial states $S T S(a)$; step transition system $S T S_{q_{2}}(b)$; and Petri net solving $S T S_{q_{1}}(c)$.

\section{Reversing steps}

The reverse action of an action $a$ in a step transition system $S T S$ or a PT-net $N$ will be denoted by $\bar{a}$. Intuitively, $\bar{a}$ cancels the effect of $a$ which corresponds to $a+\bar{a} \bowtie_{S T S} \varnothing$ and $\operatorname{eff}_{N}(a)+\operatorname{eff}_{N}(\bar{a})=0$, respectively.

We consider four ways of modifying step transition systems to capture the effect of reversing actions. In the first three, each action $a$ has a unique reverse action $\bar{a}$. Moreover, the reverse $\bar{\alpha}$ of a multiset $\alpha$ of actions is obtained by replacing each action occurrence in $\alpha$ by its reverse. In the fourth one, an action $a$ has possibly multiple unique indexed reverse actions $\bar{a}_{\langle i d x\rangle}$. The indexfree version noidx $(\alpha)$ of a multiset $\alpha$ is obtained by replacing each $\bar{a}_{\langle i d x\rangle}$ in $\alpha$ by $\bar{a}$. For example, $\operatorname{noidx}\left(\left(\bar{a}_{\langle 7\rangle} \bar{b}_{\langle s, w\rangle} \bar{b} \bar{a}_{\langle f\rangle}\right)\right)=(\bar{a} \bar{b} \bar{b} \bar{a})=\overline{(a b b a)}$.

In the domain of step transition systems, reversing is introduced at the behavioural level. The direct/set/mixed reverse of a CEST-system $S T S=\left(S, T, \rightarrow, s_{0}\right)$ is respectively given by:

$$
\begin{aligned}
& S T S^{\text {rev }}=\left(S, T \uplus \bar{T}, \rightarrow \cup \rightarrow_{\text {rev }}, s_{0}\right) \text { with } \rightarrow_{\text {rev }}=\left\{(s \oplus \alpha, \bar{\alpha}, s) \mid s \stackrel{\alpha}{\rightarrow}_{S T S}\right\} \\
& S T S^{\text {srev }}=\left(S, T \uplus \bar{T}, \rightarrow \cup \rightarrow_{\text {srev }}, s_{0}\right) \text { with } \rightarrow_{\text {srev }}=\left\{(s \oplus \alpha, \bar{\alpha}, s) \mid s \stackrel{\alpha}{\rightarrow}_{S T S} \wedge \operatorname{supp}(\alpha)=\alpha\right\} \\
& S T S^{\text {mrev }}=\left(S, T \uplus \bar{T}, \rightarrow_{\text {mrev }}, s_{0}\right) \quad \text { with } \rightarrow_{\text {mrev }}=\left\{(s \oplus \alpha, \bar{\alpha}+\beta, s \oplus \beta) \mid s \stackrel{\alpha+\beta}{\longrightarrow}_{S T S}\right\} .
\end{aligned}
$$

That is, $\rightarrow_{\text {rev }}$ reverses all the (original) steps, $\rightarrow_{\text {srev }}$ only reverses the steps that are sets, and $\rightarrow_{\text {mrev }}$ introduces partial reverses with mixed steps, including both the original and reverse actions. Figure 2 illustrates mixed reversing. Note that $s \oplus \alpha$ and $s \oplus \beta$ are states in $S T S$ due to $S E Q$ and $C E$.

In the domain of PT-nets, reversing is introduced structurally rather than behaviourally, by adding reverses at the level of actions: 


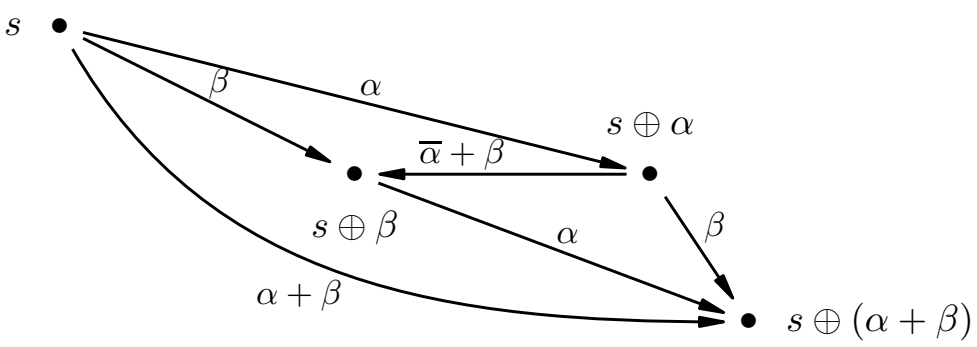

Figure 2. A mixed reverse transition $s \oplus \alpha \stackrel{\bar{\alpha}+\beta}{\longrightarrow}$ mrev $s \oplus \beta$ derived from $s \stackrel{\alpha+\beta}{\longrightarrow}$ STS.

A split reverse of $S T S$ is a step transition system $S T S^{\text {split }}=\left(S, T \uplus T^{\prime}, \rightarrow^{\prime}, s_{0}\right)$ satisfying SEQ and such that $T \cap \operatorname{noidx}\left(T^{\prime}\right)=\varnothing$ and noidx $\left(S T S^{\text {split }}\right)=S T S^{\text {rev }}$, where noidx $\left(S T S^{\text {split }}\right)=$ $\left(S, T \cup \operatorname{noidx}\left(T^{\prime}\right),\left\{\left(s, \operatorname{noidx}(\alpha), s^{\prime}\right) \mid\left(s, \alpha, s^{\prime}\right) \in \rightarrow^{\prime}\right\}, s_{0}\right)$ is the step transition system obtained from $S T S$ by replacing each occurrence of an indexed reverse action $\bar{a}_{\langle i d x\rangle}$ by $\bar{a}$. That is, $\rightarrow^{\prime}$ introduces split reverses allowing one or more reverses of a step, possibly using different reverses of the same action when reversing a step that contains its multiple copies.

- A PT-net $N$ with reverses is such that, for each original action $a$, there is a reverse action $\bar{a}$ such that $\operatorname{eff}_{N}(\bar{a})=-\operatorname{eff}_{N}(a)$.

- A PT-net $N$ with strict reverses is such that, for each original action $a$, there is a reverse action $\bar{a}$ such that $\operatorname{pre}_{N}(\bar{a})=\operatorname{post}_{N}(a)$ and $\operatorname{post}_{N}(\bar{a})=\operatorname{pre}_{N}(a)$.

- A PT-net $N$ with split reverses is such that, for each original action $a$, there is at least one indexed reverse action $\bar{a}_{\langle i d x\rangle}$ such that $\operatorname{eff}_{N}\left(\bar{a}_{\langle i d x\rangle}\right)=-\operatorname{eff}_{N}(a)$.

A key problem which then arises is that of characterising relationships between statically defined reversing of PT-nets and the behavioural reversing of their concurrent reachability graphs. In the rest of this paper, we will address this problem by providing both negative and positive results. First, however, we show basic properties of the reversed step transition systems. In particular, that all such step transition systems are CEST-systems, and that the solvability of a reversed step transition system implies the solvability of the original step transition system.

Theorem 3.1. Let $S T S$ be a CEST-system, and $S T S^{\text {split }}$ be any of its split reverses.

1. $S T S$ STS $S^{\text {srev }}$ $S T S^{r e v}$ $S T S^{m r e v}$ and $S T S$ STS $S^{\text {split }}$.

2. STS ${ }^{m r e v}, S T S^{\text {srev }}, S T S^{r e v}$, and $S T S^{\text {split }}$ are CEST-systems.

3. If any step transition system among $S T S^{m r e v}, S T S^{s r e v}, S T S^{r e v}$, and $S T S^{s p l i t}$ is solvable, then $S T S$ is also solvable.

\section{Proof:}

Let $S T S=\left(S, T, \rightarrow, s_{0}\right)$ and $S T S^{\prime}$ be any step transition system among $S T S^{m r e v}, S T S^{\text {srev }}, S T S^{r e v}$, and $S T S^{\text {split }}$. We start with an auxiliary result. 
Lemma 3.2. Let $\alpha, \beta, \gamma, \delta \in \operatorname{mult}(T)$.

1. $s \stackrel{\alpha}{\longrightarrow}$ STS mrev $s^{\prime}$ iff $s \stackrel{\alpha}{\longrightarrow}$ STS rev $s^{\prime}$ iff $s \stackrel{\alpha}{\longrightarrow}$ STS split $s^{\prime}$ iff $s \stackrel{\alpha}{\longrightarrow}$ STS $s^{\prime}$.

2. $s \stackrel{\bar{\alpha}}{\longrightarrow}$ STS ${ }^{\text {mrev }} s^{\prime}$ iff $s \stackrel{\bar{\alpha}}{\longrightarrow}$ STS $S^{\text {rev }} s^{\prime}$.

\section{Proof:}

[Lemma 3.2] (1) The second and third equivalences are obvious, so we only show the first one.

$(\Longrightarrow)$ Suppose that $s \stackrel{\alpha}{\longrightarrow} S T S^{m r e v} s^{\prime}$. Then, by the definition of $S T S^{m r e v}$, there is $r \in S$ such that $r \stackrel{\varnothing+\alpha}{\longrightarrow} S T S$ and $(s=) r \oplus \varnothing \stackrel{\bar{\varnothing}+\alpha}{\longrightarrow} S T S^{m r e v} r \oplus \alpha\left(=s^{\prime}\right)$. By Proposition 2.1(2), $s=r$. Hence, by Proposition 2.1(2), $r \oplus \alpha=s \oplus \alpha=s^{\prime}$. As a result, $s \stackrel{\alpha}{\longrightarrow} S T S s^{\prime}$.

$(\Longleftarrow)$ Suppose that $s \stackrel{\alpha}{\longrightarrow} S T S s^{\prime}$. Then $s \stackrel{\varnothing+\alpha}{\longrightarrow} S T S$ and so, by the definition of $S T S^{m r e v}$, $s \oplus \varnothing \stackrel{\bar{\varnothing}+\alpha}{\longrightarrow}_{S T S^{m r e v}} s \oplus \alpha$. By Proposition 2.1 (1), $s^{\prime}=s \oplus \alpha$, and, by Proposition 2.1(2), $s=s \oplus \varnothing$. Hence $s \stackrel{\alpha}{\longrightarrow}$ STS mrev $s^{\prime}$.

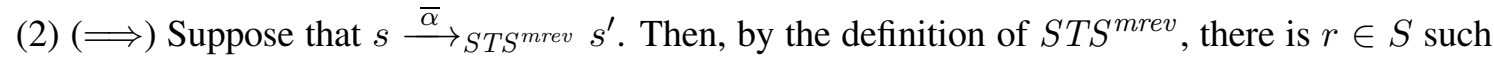
that $(s=) r \oplus \alpha \stackrel{\bar{\alpha}+\varnothing}{\longrightarrow} S T S^{m r e v} r \oplus \varnothing\left(=s^{\prime}\right)$ and $r \stackrel{\alpha+\varnothing}{\longrightarrow} S T S$. By Proposition 2.1 (2), $s^{\prime}=r$. Hence $s^{\prime} \stackrel{\alpha}{\longrightarrow} S T S$ s. Thus, by the definition of $S T S^{r e v}, s \stackrel{\bar{\alpha}}{\longrightarrow} S T S^{r e v} s^{\prime}$.

$(\Longleftarrow)$ Suppose that $s \stackrel{\bar{\alpha}}{\longrightarrow} S T S^{r e v} s^{\prime}$. Then, by the definition of $S T S^{r e v}, s^{\prime} \stackrel{\alpha+\varnothing}{\longrightarrow} S T S s$. Hence, by definition of $S T S^{m r e v}, s^{\prime} \oplus \alpha \stackrel{\bar{\alpha}+\varnothing}{\longrightarrow} S T S^{m r e v} s^{\prime} \oplus \varnothing$. By Proposition $2.1(1), s=s^{\prime} \oplus \alpha$, and, by Proposition 2.1(2), $s^{\prime}=s^{\prime} \oplus \varnothing$. Hence $s \stackrel{\alpha}{\longrightarrow} S T S^{\text {mrev }} s^{\prime}$.

(1) Follows directly from the definitions and Lemma 3.2 $(1,2)$.

(2) We discuss in turn the four properties defining CEST-systems.

(EL and REA) Follow directly from part (1) and the fact that $S T S$ satisfies $E L$ and REA.

(SEQ) For $S T S^{\text {srev }}, S T S^{r e v}$, and $S T S^{\text {split }}, S E Q$ holds directly from the definitions. To show $S E Q$ for $S T S^{m r e v}$, suppose that:

$$
s \stackrel{\alpha_{1}+\alpha_{2}+\beta_{1}+\beta_{2}}{\longrightarrow} S T S \text { and } s \oplus\left(\alpha_{1}+\alpha_{2}\right) \stackrel{\bar{\alpha}_{1}+\bar{\alpha}_{2}+\beta_{1}+\beta_{2}}{\longrightarrow} \text { STS } S^{\text {mrev }} s \oplus\left(\beta_{1}+\beta_{2}\right) .
$$

Then, by $S E Q$ for $S T S$, we have $s \oplus \alpha_{2} \stackrel{\alpha_{1}+\beta_{1}}{\longrightarrow} S T S$ and $s \oplus \beta_{1} \stackrel{\alpha_{2}+\beta_{2}}{\longrightarrow} S T S$. Hence, by the definition of $S T S^{m r e v}$,

$$
\begin{array}{lll}
\left(s \oplus \alpha_{2}\right) \oplus \alpha_{1} & \stackrel{\bar{\alpha}_{1}+\beta_{1}}{\longrightarrow} \text { STS }_{\text {mrev }} & \left(s \oplus \alpha_{2}\right) \oplus \beta_{1} \\
\left(s \oplus \beta_{1}\right) \oplus \alpha_{2} & \stackrel{\bar{\alpha}_{2}+\beta_{2}}{\longrightarrow} S_{S T} S^{\text {mrev }} & \left(s \oplus \beta_{1}\right) \oplus \beta_{2} .
\end{array}
$$

Moreover, by Proposition 2.2, we have:

$$
\begin{aligned}
& s \oplus\left(\alpha_{2}+\alpha_{1}\right)=\left(s \oplus \alpha_{2}\right) \oplus \alpha_{1} \\
& \left(s \oplus \beta_{1}\right) \oplus \beta_{2}=s \oplus\left(\beta_{1}+\beta_{2}\right) \\
& \left(s \oplus \alpha_{2}\right) \oplus \beta_{1}=s \oplus\left(\alpha_{2}+\beta_{1}\right)=\left(s \oplus \beta_{1}\right) \oplus \alpha_{2} .
\end{aligned}
$$

Hence, $s \oplus\left(\alpha_{1}+\alpha_{2}\right) \stackrel{\bar{\alpha}_{1}+\beta_{1}}{\longrightarrow}$ STS $S^{\text {mrev }} s \oplus\left(\alpha_{2}+\beta_{1}\right) \stackrel{\bar{\alpha}_{2}+\beta_{2}}{\longrightarrow} S_{S T S^{\text {mrev }}} s \oplus\left(\beta_{1}+\beta_{2}\right)$. 
(CE) We first observe that $s \stackrel{\bar{a}}{\longrightarrow} S T S^{m r e v} s^{\prime}$ implies $s^{\prime} \stackrel{\bar{a}}{\longrightarrow} S T S^{m r e v} s$, by Lemma 3.2 and the definition of $S T S^{r e v}(*)$.

We have already demonstrated that $S E Q$ holds for $S T S^{\prime}$. Hence, by Propositions 2.3, it suffices to show that $C E$ holds for $\left(S T S^{\prime}\right)^{s e q}$.

By Propositions 2.3, we have that $S T S^{s e q}$ satisfies $C E$. Moreover, by Lemma 3.2(1) as well as the definition of $S T S^{\prime}$ and (*), $\left(S T S^{\prime}\right)^{s e q}$ can be derived by a successive application of the construction from the formulation of Proposition 2.4 (once for each reverse action and indexed reverse action). Hence, by Propositions 2.4 $\left(S T S^{\prime}\right)^{s e q}$ satisfies $C E$.

(3) Let $N^{\prime}=\left(P, T^{\prime}, F, M_{0}\right)$ be a PT-net such that $S T S^{\prime} \simeq_{\psi} C R G_{N^{\prime}}$. We will show that $S T S \simeq_{\psi}$ $C R G_{N}$, where $N=\left.N^{\prime}\right|_{T}$. Note that the enabling and firing of steps over $T$ is exactly the same in both $N$ and $N^{\prime}(*)$.

We first observe that $\psi\left(s_{0}\right)=M_{0}$. Suppose then that $s \in S$ and $\psi(s) \in \operatorname{reach}_{N}$. To show that the executions of steps are preserved by $\psi$ in both directions, we consider two cases for $\alpha \in \operatorname{mult}(T)$.

Case 1: $s \stackrel{\alpha}{\longrightarrow}$ STS $s^{\prime}$. Then, by part (1), $s \stackrel{\alpha}{\longrightarrow}{ }_{S T S^{\prime}} s^{\prime}$. Hence, by $S T S^{\prime} \simeq_{\psi} C R G_{N^{\prime}}$, we have $\psi(s) \stackrel{\alpha}{\longrightarrow} N^{\prime} \psi\left(s^{\prime}\right)$. Thus, by (*), $\psi(s) \stackrel{\alpha}{\longrightarrow}{ }_{N} \psi\left(s^{\prime}\right)$.

Case 2: $\psi(s) \stackrel{\alpha}{\longrightarrow} N$. Then, by $(*), \psi(s) \stackrel{\alpha}{\longrightarrow} N^{\prime} M$. Hence, by $S T S^{\prime} \simeq_{\psi} C R G_{N^{\prime}}$, we have $M \in \psi(S)$ and $s \stackrel{\alpha}{\longrightarrow} S T S^{\prime} \psi^{-1}(M)$. Thus, by Lemma 3.2 (1), $s \stackrel{\alpha}{\longrightarrow} S T S \psi^{-1}(M)$.

\section{Multiset and set reversibility}

The investigation of different notions of step reversibility starts with a straightforward but important negative result stating that, in the domain of PT-nets, the concept of direct reversibility — which directly generalises sequential reversibility and should be considered as the preferred way of reversing step transition systems - cannot handle steps which are true multisets.

Proposition 4.1. Let $S T S$ be a CEST-system which is not a set transition system. Then $S T S^{r e v}$ is not solvable.

\section{Proof:}

[Figure 3 $(a)$ illustrates the idea of the proof.] Let $S T S=\left(S, T, \rightarrow, s_{0}\right)$. Suppose that $S T S^{r e v}$ is solvable. Then there is a PT-net $N$ such that $S T S^{r e v} \simeq_{\psi} C R G_{N}(*)$. As $S T S$ is not a set transition system, there are $v \in S$ and $\alpha \in \operatorname{mult}(T)$ such that $v \stackrel{\alpha}{\longrightarrow} S T S$ and $(a a) \leq \alpha$, for some $a \in T$.

By SEQ for $S T S$ and Theorem $3.1(1)$, there are $w, q \in S$ such that $v \stackrel{(a a)}{\longrightarrow} S T S^{r e v} \quad w$ and $v \stackrel{a}{\longrightarrow} S T S^{r e v} q(* *)$. Hence, by the definition of $S T S^{r e v}, w \stackrel{(\overline{a a})}{\longrightarrow} S T S^{\text {rev }} v(* * *)$.

Let $M_{s}=\psi(s)$, for $s \in\{v, w, q\}$. By the definition of $S T S^{r e v}$ and $\left(^{*}\right)$, the step $\beta=(a \bar{a})$ is not enabled at $M_{q}$. Hence, there is a place $p$ of $N$ such that $M_{q}(p)<\operatorname{pre}_{N}(\beta)(p)(\dagger)$. On the other hand, by $(* *)$ and $(* * *)$, we have:

$$
\operatorname{pre}_{N}(a a) \leq M_{v} \quad \operatorname{pre}_{N}(\overline{a a}) \leq M_{w} \quad M_{w}=M_{v}+\operatorname{eff}_{N}(a a) \quad M_{q}=M_{v}+\operatorname{eff}_{N}(a) .
$$

Thus $\operatorname{pre}_{N}(\beta)+\operatorname{pre}_{N}(\beta)=\operatorname{pre}_{N}(a a \overline{a a}) \leq M_{v}+M_{w}=M_{v}+M_{v}+$ eff $_{N}(a a)=M_{q}+M_{q}$, yielding a contradiction with $(\dagger)$. 
(a)
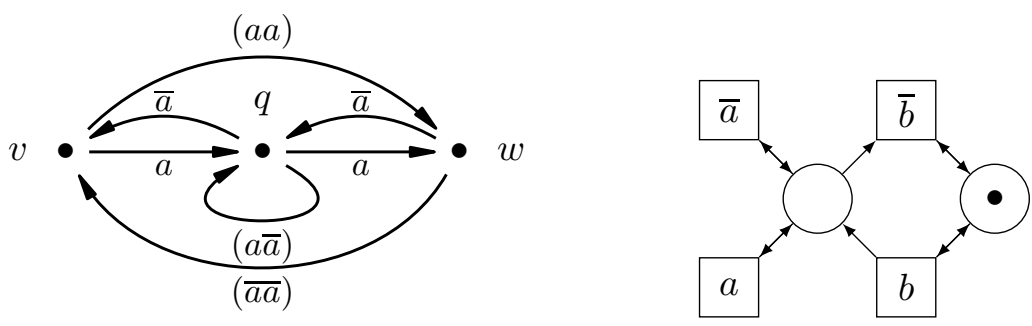

(b)

Figure 3. An illustration of the proof of Proposition 4.1 (a), and PT-net generating concurrent reachability graph which is not step-finite $(b)$.

In view of Proposition 4.1, when facing the problem of implementing a reverse of non-set step transition system $S T S$ using PT-nets, one may consider set reversibility based on $S T S^{\text {srev }}$, or mixed reversibility based on $S T S^{m r e v} 5$

Among these two options, one might prefer $S T S^{\text {srev }}$ to $S T S^{m r e v}$ as the latter introduces steps containing both the original and reverse actions. However, as the next example shows, it not always possible to 'replace' a mixed reversibility solution by a set reversibility solution.

Example 4.2. Let us consider a CEST-system $S T S=\left(\left\{s_{0}, s_{1}, \ldots\right\},\{a, b\}, \rightarrow, s_{0}\right)$ such that:

$$
s_{i} \stackrel{a^{j}}{\longrightarrow} S T S s_{i} \quad \text { and } \quad s_{i} \stackrel{b+a^{j}}{\longrightarrow} \text { STS } s_{i+1} \text { for all } i \geq 0 \text { and } j \leq i .
$$

It is straightforward to see that $S T S^{m r e v}$ is solvable by the PT-net shown in Figure $3(b)$. However, $S T S^{\text {srev }}$ is not solvable by any PT-net. If such a PT-net $N$ existed, then it would have distinct reachable markings $M_{0}, M_{1}, \ldots$ satisfying, for every $i \geq 0$ :

$$
M_{i} \stackrel{b}{\longrightarrow} N M_{i+1}(*) \quad M_{i} \stackrel{a^{i}}{\longrightarrow} N M_{i}(* *) \quad M_{i} \stackrel{\bar{a}}{\longrightarrow} M_{N} M_{i}(* * *) \quad \neg M_{i} \stackrel{(a \bar{a})}{\longrightarrow} N(\dagger) .
$$

We now observe that $M_{0} \leq M_{1} \leq \cdots$ due to $(*)$. Hence, there is a place $p$ such that $\operatorname{pre}_{N}(a \bar{a})(p)>$ $M_{0}(p)=M_{1}(p)=\cdots(\ddagger)$, due to $(\dagger)$ and the finiteness of $N$. On the other hand, $\operatorname{pre}_{N}(\bar{a})(p) \leq$ $M_{0}(p)=M_{1}(p)=\cdots$ due to $(* * *)$, and $\operatorname{pre}_{N}(a)(p)=0$ due to (**) and ( $)$. As a result, $\operatorname{pre}_{N}(a \bar{a})(p) \leq M_{0}(p)$, yielding a contradiction with $(\ddagger)$.

Example 4.2 demonstrated that there are step transition systems which can be treated using mixed reversibility, but not using set reversibility. What is more, the example worked because the step transition system considered was not step-finite. As the next result shows, that was the only reason why set reversibility failed to hold.

Theorem 4.3. Let $S T S$ be a CEST-system such that $S T S^{m r e v}$ is solvable. Then $S T S^{\text {srev }}$ is solvable if and only if $S T S$ is step-finite.

\footnotetext{
${ }^{5}$ We will discuss split reversibility separately in Section 7
} 


\section{Proof:}

Let $S T S=\left(S, T, \rightarrow, s_{0}\right)$.

$(\Longrightarrow)$ Suppose that $S T S^{\text {srev }}$ is solvable by a PT-net $N=\left(P, T \cup \bar{T}, F, M_{0}\right)$, and that $S T S$ is not step-finite. By the finiteness of $P$ and $T$ as well as $S E Q$ for $S T S$, there is $a \in T$ and reachable markings $M_{1} \leq M_{2} \leq \ldots$ such that $M_{i} \stackrel{a^{i}}{\longrightarrow}$, for every $i \geq 1$. Hence, by $S E Q$ for $C R G_{N}$, there is a marking $M_{i}^{\prime}$ such that $M_{i} \stackrel{a}{\longrightarrow} M_{i}^{\prime}$ and $M_{i}^{\prime} \stackrel{a^{i-1}}{\longrightarrow}{ }_{N}(*)$, for every $i \geq 1$. As a result, $M_{i}^{\prime} \stackrel{a}{\longrightarrow} N$ and $M_{i}^{\prime} \stackrel{\bar{a}}{\longrightarrow} N(* *)$, for every $i \geq 2$.

We now observe that $(M=) M_{m+2}^{\prime} \stackrel{(a \bar{a})}{\longrightarrow}$, where $m=\max \{F(p, \bar{a}) \mid p \in P\}$. Indeed, otherwise there is $p \in P$ such that $M(p)<F(p, a)+F(p, \bar{a}) \leq F(p, a)+m(\dagger)$. On the other hand, by (**), $M(p) \geq F(p, a)$ and $M(p) \geq F(p, \bar{a})$. Hence, it must be the case that $F(p, a)>0$. Thus, by $(*), M(p) \geq(m+1) \cdot F(a, p)=m+F(a, p)$, contradicting $(\dagger)$. As a result, $M \stackrel{(a \bar{a})}{\longrightarrow}{ }_{N}$, yielding a contradiction with our initial assumption.

( $\Longleftarrow$ ) If $S T S$ is step-finite, then there is $k \geq 1$ such that $|\alpha| \leq k$, whenever $s \stackrel{\alpha}{\longrightarrow} S T S$. Moreover, since $S T S^{m r e v}$ is solvable, there exists a PT-net $N=\left(P, T \cup \bar{T}, F, M_{0}\right)$ such that $S T S^{\text {mrev }} \simeq_{\psi}$ $C R G_{N}$. We then modify $N$, by adding to $P$ a set of fresh places $P^{\prime}=\left\{p_{a b} \mid a \in T \wedge b \in \bar{T}\right\}$. Each $p_{a b}$ is such that $M_{0}\left(p_{a b}\right)=k$ and has four non-zero connections, $F\left(a, p_{a b}\right)=F\left(p_{a b}, a\right)=1$ and $F\left(b, p_{a b}\right)=F\left(p_{a b}, b\right)=k$. For the resulting PT-net $N^{\prime}$, we have $S T S^{\text {srev }} \simeq_{\psi^{\prime}} C R G_{N^{\prime}}$, where $\psi^{\prime}(s)=\psi(s)+\sum_{p \in P^{\prime}} p^{k}$, for every $s \in S$.

We have therefore obtained a full characterisation of step transition systems for which mixed reversibility solutions can be replaced by set reversibility solutions. In addition, the second part of the proof of Theorem 4.3 provides a straightforward construction achieving this.

A direct corollary of the last result is that for a set step transition system it is always possible to replace a mixed reversibility solution by a set reversibility solution.

Theorem 4.4. Let $S T S$ be a set CEST-system. If $S T S^{m r e v}$ is solvable, then $S T S^{r e v}$ is also solvable.

\section{Proof:}

As a set CEST-system, $S T S$ is step-finite and $S T S^{r e v}=S T S^{\text {srev }}$. Hence the result follows from Theorem 4.3 .

A concluding observation is that all three versions of reversibility which do not involve splitting are worthy of investigation.

\section{Mixed reversibility}

In this section, we consider the problem of deciding whether the mixed reverse $S T S^{\text {mrev }}$ of a solvable step transition system $S T S$ is also solvable. A specific concern we implicitly address is the size of $S T S^{m r e v}$ which (in the finite case) can be exponentially larger than that of $S T S$. The aim is therefore to avoid dealing directly with $S T S^{m r e v}$. As shown below, this is possible as the checking of feasibility 
of mixed reversing can be replaced by checking the solvability of the original transition system, and the solvability of its reverse.

Throughout this section we make the following assumptions:

- $S T S=\left(S, T, \rightarrow, s_{0}\right)$ is a CEST-system and $R$ is a home cover of $S T S$.

- $\overline{S T S}=\left(S, \bar{T},\left\{\left(s^{\prime}, \bar{\alpha}, s\right) \mid s \stackrel{\alpha}{\longrightarrow} S T S s^{\prime}\right\}, R\right)$ is a step transition system with multiple initial states.

- $\overline{S T S}_{r}=\left(S_{r}, \bar{T}, \rightarrow_{r}, r\right)$ is a step transition system such that $r \in R, S_{r}=\{s \in S \mid r \in$ $\left.\operatorname{pred}_{S T S}(s)\right\}$, and $\rightarrow_{r}=\rightarrow \cap\left(S_{r} \times \operatorname{mult}(T) \times S_{r}\right)$.

That is, $\overline{S T S}$ is obtained by reversing each transition of $S T S$, and considering all the states in the home cover $R$ as the initial states.

Proposition 5.1. Let $r \in R$.

1. $\overline{S T S}_{r}$ is a CEST-system.

2. $s_{0} \in \bigcap_{s \in S_{r}} \operatorname{pred}_{S T S}(s)$.

3. $S=\bigcup_{r \in R} S_{r}$.

\section{Proof:}

(1) The only non-trivial property to show is $C E$. For every $\mathcal{A}$-vector $\alpha$ with support $\bar{T}$, let $\widehat{\alpha}$ be the $\mathcal{A}$-vector with support $T$ such that $\widehat{\alpha}(a)=-\alpha(\bar{a})$, for every $a \in T$.

We first observe that, for every $\pi \in \operatorname{paths}_{\operatorname{STS}_{r}}\left(s, s^{\prime}\right)$, there is $\pi^{\prime} \in \operatorname{paths}_{S T S}\left(s, s^{\prime}\right)$ such that $\operatorname{sign}\left(\pi^{\prime}\right)=\widehat{\operatorname{sign}(\pi)}(*)$. Hence, we also have that $\alpha \bowtie_{S T S} \beta$ implies $\widehat{\alpha} \bowtie_{S T S} \widehat{\beta}$, for all $\mathcal{A}$-vectors $\alpha$ and $\beta$ with support $\bar{T}(* *)$. Thus, $\overline{S T S}_{r}$ satisfies $C E$ by (*) and (**).

(2) Follows from the fact that $S T S$ satisfies REA.

(3) Follows from the fact that $R$ is a home cover.

Theorem 5.2. $S T S^{m r e v}$ is solvable if and only if both $S T S$ and $\overline{S T S}$ are solvable.

\section{Proof:}

( $\Longrightarrow$ ) By Theorem 3.1(3), STS is solvable. To show that $\overline{S T S}$ is solvable, suppose that $N=$ $\left(P, T, F, M_{0}\right)$ is a PT-net such that $S T S^{m r e v} \simeq_{\psi} C R G_{N}$. We will show that $\overline{S T S}_{r} \simeq_{\psi \mid S_{r}} C R G_{N_{r}}$, where, for every $r \in R, N_{r}$ is the PT-net $\left.N\right|_{T}$ with the initial marking set to $\psi(r)$. Note that the enabling and firing of steps over $\bar{T}$ is exactly the same in both $N$ and $N_{r}$ (*).

We first observe that the initial states of $\overline{S T S}_{r}$ and $C R G_{N_{r}}$ are related by $\psi$. Suppose then that $s \in S_{r}$ is such that $\psi(s) \in$ reach $_{N_{r}}$. To show that the executions of steps are preserved by $\psi$ in both directions, we consider two cases, where $\alpha \in \operatorname{mult}(T)$.

Case 1.1: $s \stackrel{\bar{\alpha}}{\longrightarrow} \overline{S T S}_{r} s^{\prime}$. Then $s \stackrel{\bar{\alpha}}{\longrightarrow}$ STS $S^{r e v} s^{\prime}$ and so, by Lemma $3.2(2), s \stackrel{\bar{\alpha}}{\longrightarrow}$ STS mrev $s^{\prime}$. Hence, by $S T S^{m r e v} \simeq_{\psi} C R G_{N}$, we have $\psi(s) \stackrel{\bar{\alpha}}{\longrightarrow} \psi\left(s^{\prime}\right)$. Thus, by $(*), \psi(s) \stackrel{\bar{\alpha}}{\longrightarrow} N_{r} \psi\left(s^{\prime}\right)$. 
Case 1.2: $\psi(s) \stackrel{\bar{\alpha}}{\longrightarrow} N_{r} M$. Then, by $(*), \psi(s) \stackrel{\bar{\alpha}}{\longrightarrow} N M$. Hence, by $S T S^{\text {mrev }} \simeq_{\psi} C R G_{N}$, we have $M \in \psi(S)$ and $s \stackrel{\bar{\alpha}}{\longrightarrow} S T S^{m r e v} \psi^{-1}(M)$. Thus, by Lemma 3.2 (2), $s \stackrel{\bar{\alpha}}{\longrightarrow}$ STS ${ }^{r e v} \psi^{-1}(M)$. Hence $s \stackrel{\bar{\alpha}}{\longrightarrow} \overline{S T S}_{r} \psi^{-1}(M)$.

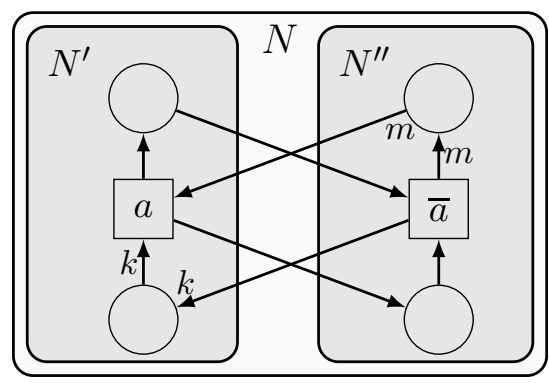

Figure 4. An illustration of the second part of the proof of Theorem 5.2

$\left(\Longleftarrow\right.$ ) Since $S T S$ is solvable, there is a PT-net $N^{\prime}=\left(P^{\prime}, T, F^{\prime}, M_{0}^{\prime}\right)$ such that $S T S \simeq_{\psi^{\prime}} C R G_{N^{\prime}}$. (Note that $\psi^{\prime}\left(s_{0}\right)=M_{0}^{\prime}$.) Moreover, since $\overline{S T S}$ is solvable, there is an umarked PT-net $N^{\prime \prime}=$ $\left(P^{\prime \prime}, \bar{T}, F^{\prime \prime}\right)$ and a mapping $\psi^{\prime \prime}: S \rightarrow \operatorname{mult}\left(P^{\prime \prime}\right)$ such that $\overline{S T S}_{r} \simeq_{\left.\psi^{\prime \prime}\right|_{S_{r}}} C R G_{N_{r}}$, where $N_{r}=$ $\left(P^{\prime \prime}, \bar{T}, F^{\prime \prime}, M_{r}\right)$ and $M_{r}=\psi^{\prime \prime}(r)$, for every $r \in R$. Clearly, we may assume that $P^{\prime} \cap P^{\prime \prime}=\varnothing$ as the identities of places play no role in the solvability problems of $S T S$ and $\overline{S T S}$.

Let $N=\left(P^{\prime} \cup P^{\prime \prime}, T \cup \bar{T}, F, M_{0}\right)$ be the PT-net with strict reverses (illustrated in Figure 4) such that $M_{0}=M_{0}^{\prime} \sqcup \psi^{\prime \prime}\left(s_{0}\right)=\psi^{\prime}\left(s_{0}\right) \sqcup \psi^{\prime \prime}\left(s_{0}\right)$ and, for every $a \in T$ :

$$
\begin{array}{ll}
\operatorname{pre}_{N}(a)=\operatorname{pre}_{N^{\prime}}(a) \sqcup \operatorname{post}_{N^{\prime \prime}}(\bar{a}) & \operatorname{post}_{N}(a)=\operatorname{post}_{N^{\prime}}(a) \sqcup \operatorname{pre}_{N^{\prime \prime}}(\bar{a}) \\
\operatorname{pre}_{N}(\bar{a})=\operatorname{pre}_{N^{\prime \prime}}(\bar{a}) \sqcup \operatorname{post}_{N^{\prime}}(a) & \operatorname{post}_{N}(\bar{a})=\operatorname{post}_{N^{\prime \prime}}(\bar{a}) \sqcup \operatorname{pre}_{N^{\prime}}(a) .
\end{array}
$$

Let $\psi$ be a mapping with the domain $S$ which, for every $s \in S$, returns $\psi^{\prime}(s) \sqcup \psi^{\prime \prime}(s)$. Note that $\psi$ is well-defined due to Lemma5.1 (3) and $\psi\left(s_{0}\right)=M_{0}$.

Lemma 5.3. Let $S T S^{\prime}$ be $C R G_{N}$ with all the transitions labelled by steps of the form $\alpha+\bar{\beta}$, for $\alpha, \beta \neq \varnothing$, deleted.

1. $S T S^{r e v} \simeq_{\psi} S T S^{\prime}$.

2. $S T S^{\prime}$ satisfies REA.

3. $\psi(s \oplus \alpha)=\psi(s)+\operatorname{eff}_{N}(\alpha)$, for all $s \stackrel{\alpha}{\longrightarrow} S T S$.

\section{Proof:}

[Lemma 5.3] (1) We observe that the initial states of $S T S^{r e v}$ and $S T S^{\prime}$ are related by $\psi$. Suppose now that $s \in S$ and $\psi(s) \in \operatorname{reach}_{N}$. To show that the executions of steps are preserved by $\psi$ in both directions, we consider four cases, where $\alpha \in \operatorname{mult}(T)$. 
Case 2.1: $s \stackrel{\alpha}{\longrightarrow}_{S T S^{\text {rev }}} s^{\prime}$. Then, by $S T S \simeq_{\psi^{\prime}} C R G_{N^{\prime}}$, we have $\psi^{\prime}(s) \stackrel{\alpha}{\longrightarrow}_{N^{\prime}} \psi^{\prime}\left(s^{\prime}\right)$ and $\psi^{\prime}(s) \geq$ $\operatorname{pre}_{N^{\prime}}(\alpha)$. Moreover, $s^{\prime} \stackrel{\bar{\alpha}}{\longrightarrow} S T S^{\text {rev }} s$. Hence, by Lemma 5.1 (3), there is $r \in R$ such that $s^{\prime} \stackrel{\bar{\alpha}}{\longrightarrow} \overline{S T S}_{r} s$. Thus, by $\overline{S T S}_{r} \simeq_{\left.\psi^{\prime \prime}\right|_{S_{r}}} C R G_{N_{r}}$, we have $\psi^{\prime \prime}\left(s^{\prime}\right) \stackrel{\bar{\alpha}}{\longrightarrow}_{N^{\prime \prime}} \psi^{\prime \prime}(s)$ and $\psi^{\prime \prime}(s) \geq \operatorname{post}_{N^{\prime \prime}}(\bar{\alpha})$. Hence, by Eq.(2):

$$
\psi(s)=\left(\psi^{\prime}(s) \sqcup \psi^{\prime \prime}(s)\right) \geq\left(\operatorname{pre}_{N^{\prime}}(\alpha) \sqcup \operatorname{post}_{N^{\prime \prime}}(\bar{\alpha})\right)=\operatorname{pre}_{N}(\alpha) .
$$

As a result, $\psi(s) \stackrel{\alpha}{\longrightarrow} N \psi(s)+\operatorname{eff}_{N}(\alpha)$. Hence $\psi(s) \stackrel{\alpha}{\longrightarrow}{ }_{N} \psi\left(s^{\prime}\right)$ as we have, by Eq.(2):

$$
\begin{aligned}
\psi(s)+\operatorname{eff}_{N}(\alpha) & =\left(\psi^{\prime}(s) \sqcup \psi^{\prime \prime}(s)\right)+\operatorname{post}_{N}(\alpha)-\operatorname{pre}_{N}(\alpha) \\
& =\left(\psi^{\prime}(s) \sqcup \psi^{\prime \prime}(s)\right)+\left(\operatorname{post}_{N^{\prime}}(\alpha) \sqcup \operatorname{pre}_{N^{\prime \prime}}(\bar{\alpha})\right)-\left(\operatorname{pre}_{N^{\prime}}(\alpha) \sqcup \operatorname{post}_{N^{\prime \prime}}(\bar{\alpha})\right) \\
& =\left(\psi^{\prime}(s)+\operatorname{eff}_{N^{\prime}}(\alpha)\right) \sqcup\left(\psi^{\prime \prime}(s)-\operatorname{eff}_{N^{\prime \prime}}(\bar{\alpha})\right) \\
& =\psi^{\prime}\left(s^{\prime}\right) \sqcup \psi^{\prime \prime}\left(s^{\prime}\right)=\psi\left(s^{\prime}\right) .
\end{aligned}
$$

Case 2.2: $s \stackrel{\bar{\alpha}}{\longrightarrow} S T S^{r e v} s^{\prime}$. Then $s^{\prime} \stackrel{\alpha}{\longrightarrow}_{S T S^{r e v}} s$ and so, by Case $2.1, \psi\left(s^{\prime}\right) \stackrel{\alpha}{\longrightarrow} N \psi(s)$. Hence, since $N$ is a PT-net with strict reverses, $\psi(s) \stackrel{\bar{\alpha}}{\longrightarrow}{ }_{N} \psi\left(s^{\prime}\right)$.

Case 2.3: $\psi(s) \stackrel{\alpha}{\longrightarrow} N$. Then, by Eq.(2), we have:

$$
\begin{gathered}
\psi^{\prime}(s) \sqcup \psi^{\prime \prime}(s)=\psi(s) \geq \operatorname{pre}_{N}(\alpha)=\operatorname{pre}_{N^{\prime}}(\alpha) \sqcup \operatorname{post}_{N^{\prime \prime}}(\bar{\alpha}) \\
M=\psi(s)+\operatorname{eff}_{N}(\alpha)=\left(\psi^{\prime}(s) \sqcup \psi^{\prime \prime}(s)\right)+\left(\operatorname{post}_{N^{\prime}}(\alpha) \sqcup \operatorname{pre}_{N^{\prime \prime}}(\bar{\alpha})\right)-\left(\operatorname{pre}_{N^{\prime}}(\alpha) \sqcup \operatorname{post}_{N^{\prime \prime}}(\bar{\alpha})\right) .
\end{gathered}
$$

Hence, by $P^{\prime} \cap P^{\prime \prime}=\varnothing, \psi^{\prime}(s) \geq \operatorname{pre}_{N^{\prime}}(\alpha)$ and $\psi^{\prime \prime}(s) \geq \operatorname{post}_{N^{\prime \prime}}(\bar{\alpha})$ as well as:

$$
\left.M\right|_{P^{\prime}}=\psi^{\prime}(s)+\operatorname{eff}_{N^{\prime}}(\alpha) \quad \text { and }\left.\quad M\right|_{P^{\prime \prime}}=\psi^{\prime \prime}(s)-\operatorname{eff}_{N^{\prime \prime}}(\bar{\alpha}) .
$$

Thus $\left.\psi^{\prime}(s) \stackrel{\alpha}{\longrightarrow} N_{N^{\prime}} M\right|_{P^{\prime}}$. Hence, by $S T S \simeq_{\psi^{\prime}} C R G_{N^{\prime}}$, we obtain $\left.M\right|_{P^{\prime}} \in \psi^{\prime}(S)$ and $s \stackrel{\alpha}{\longrightarrow}{ }_{S T S^{r e v}} s^{\prime}$, where $\psi^{\prime}\left(s^{\prime}\right)=\left.M\right|_{P^{\prime}}$. We still need to show that $\psi\left(s^{\prime}\right)=M$. This follows from $\psi^{\prime \prime}\left(s^{\prime}\right)=\left.M\right|_{P^{\prime \prime}}$. Indeed, we have $s^{\prime} \stackrel{\bar{\alpha}}{\longrightarrow} S T S^{r e v} s$ and so, by Lemma 5.1(3), there is $r \in R$ such that $s^{\prime} \in S_{r}$. Now, by $\overline{S T S}_{r} \simeq_{\left.\psi^{\prime \prime}\right|_{S r}} C R G_{N_{r}}, \psi^{\prime \prime}\left(s^{\prime}\right) \stackrel{\bar{\alpha}}{\longrightarrow} N^{\prime \prime} \psi^{\prime \prime}(s)$, which means that $\psi^{\prime \prime}\left(s^{\prime}\right)=\psi^{\prime \prime}(s)-\operatorname{eff}_{N^{\prime \prime}}(\bar{\alpha})=\left.M\right|_{P^{\prime \prime}}$.

Case 2.4: $\psi(s) \stackrel{\bar{\alpha}}{\longrightarrow} N$. Then, by Eq.(2), we have:

$$
\begin{gathered}
\psi^{\prime}(s) \sqcup \psi^{\prime \prime}(s)=\psi(s) \geq \operatorname{pre}_{N}(\bar{\alpha})=\operatorname{pre}_{N^{\prime \prime}}(\bar{\alpha}) \sqcup \operatorname{post}_{N^{\prime}}(\alpha) \\
M=\left(\psi^{\prime}(s) \sqcup \psi^{\prime \prime}(s)\right)+\left(\operatorname{post}_{N^{\prime \prime}}(\bar{\alpha}) \sqcup \operatorname{pre}_{N^{\prime}}(\alpha)\right)-\left(\operatorname{pre}_{N^{\prime \prime}}(\bar{\alpha}) \sqcup \operatorname{post}_{N^{\prime}}(\alpha)\right) .
\end{gathered}
$$

Hence, by $P^{\prime} \cap P^{\prime \prime}=\varnothing, \psi^{\prime}(s) \geq \operatorname{post}_{N^{\prime}}(\alpha)$ and $\psi^{\prime \prime}(s) \geq \operatorname{pre}_{N^{\prime \prime}}(\bar{\alpha})$ as well as:

$$
\left.M\right|_{P^{\prime}}=\psi^{\prime}(s)-\operatorname{eff}_{N^{\prime}}(\alpha) \quad \text { and }\left.\quad M\right|_{P^{\prime \prime}}=\psi^{\prime \prime}(s)+\operatorname{eff}_{N^{\prime \prime}}(\bar{\alpha}) .
$$

Thus $\left.\psi^{\prime \prime}(s) \stackrel{\bar{\alpha}}{\longrightarrow} N^{\prime \prime} M\right|_{P^{\prime \prime}}$. Hence, by Lemma 5.1(3), there is $r \in R$ such that $s \in S_{r}$. Thus, by $\overline{S T S}_{r} \simeq_{\left.\psi^{\prime \prime}\right|_{S r}} C R G_{N_{r}},\left.M\right|_{P^{\prime \prime}} \in \psi^{\prime \prime}(S)$ and $s \stackrel{\bar{\alpha}}{\longrightarrow} S T S^{\text {rev }} s^{\prime}$, where $\psi^{\prime \prime}\left(s^{\prime}\right)=\left.M\right|_{P^{\prime \prime}}$. We still need to show that $\psi(s)=M$. This follows from $\psi^{\prime}\left(s^{\prime}\right)=\left.M\right|_{P^{\prime}}$. Indeed, we have $s^{\prime} \stackrel{\alpha}{\longrightarrow}$ STS rev $s$ and so, by $S T S \simeq_{\psi^{\prime}} C R G_{N^{\prime}}$, we obtain $\psi^{\prime}\left(s^{\prime}\right) \stackrel{\alpha}{\longrightarrow} N^{\prime} \psi^{\prime}(s)$, which means that $\psi^{\prime}\left(s^{\prime}\right)=\psi^{\prime}(s)-\operatorname{eff}_{N^{\prime}}(\alpha)=$ $\left.M\right|_{P^{\prime}}$.

(2) The modification of $C R G_{N}$ does not produce unreachable states since $C R G_{N}$ satisfies $S E Q$.

(3) Follows from part (1) and the forward determinism of $S T S$ and $C R G_{N}$. 
Returning to the proof of $S T S^{m r e v} \simeq_{\psi} C R G_{N}$, suppose that $s \in S$ is such that $\psi(s) \in \operatorname{reach}_{N}$ and consider two cases, where $\alpha, \beta \in \operatorname{mult}(T)$.

Case 3.1: $s \stackrel{\alpha+\beta}{\longrightarrow} S T S$ and $s \oplus \alpha \stackrel{\bar{\alpha}+\beta}{\longrightarrow}$ STS mrev $s \oplus \beta$. Then we have $s \stackrel{\alpha+\beta}{\longrightarrow}$ STSrev as well as:

$$
s \stackrel{\alpha}{\longrightarrow} \text { STS } s \oplus \alpha \quad s \stackrel{\beta}{\longrightarrow} \text { STS } s \oplus \beta \quad s \stackrel{\alpha}{\longrightarrow} \text { STS } \operatorname{rev} s \oplus \alpha \quad s \stackrel{\beta}{\longrightarrow} \text { STS } S^{\text {rev }} s \oplus \beta .
$$

Hence, by Lemma 5.3 $(1,3)$, we have:

$\psi(s) \stackrel{\alpha+\beta}{\longrightarrow}_{N} \quad \psi(s) \stackrel{\alpha}{\longrightarrow}_{N} \psi(s \oplus \alpha)=\psi(s)+\operatorname{eff}_{N}(\alpha) \quad \psi(s) \stackrel{\beta}{\longrightarrow}_{N} \psi(s \oplus \beta)=\psi(s)+\operatorname{eff}_{N}(\beta)$.

Thus $\psi(s) \geq \operatorname{pre}_{N}(\alpha+\beta)$, and so $\psi(s)+\operatorname{eff}_{N}(\alpha) \geq \operatorname{pre}_{N}(\alpha+\beta)+\operatorname{eff}_{N}(\alpha)=\operatorname{pre}_{N}(\bar{\alpha}+\beta)$ due to Eq.(2). Hence, again by Eq.(2):

$\psi(s \oplus \alpha)=\psi(s)+\operatorname{eff}_{N}(\alpha) \stackrel{\bar{\alpha}+\beta}{\longrightarrow}{ }_{N} \psi(s)+\operatorname{eff}_{N}(\alpha)+\operatorname{eff}_{N}(\bar{\alpha}+\beta)=\psi(s)+\operatorname{eff}_{N}(\beta)=\psi(s \oplus \beta)$.

Case 3.2: $\psi(s) \stackrel{\bar{\alpha}+\beta}{\longrightarrow} N$. Then $\psi(s) \stackrel{\bar{\alpha}}{\longrightarrow}{ }_{N} \psi(s)+\operatorname{eff}_{N}(\bar{\alpha})\left(=M^{\prime}\right)$. Hence, by Lemma 5.3 $(1)$, $s \stackrel{\bar{\alpha}}{\longrightarrow} S T S^{r e v} \psi^{-1}\left(M^{\prime}\right)\left(=s^{\prime}\right)$. Thus, by the definition of $S T S^{r e v}, s^{\prime} \stackrel{\alpha}{\longrightarrow}_{S T S} s=s^{\prime} \oplus \alpha$. We then observe that, by Eq.(2):

$$
M^{\prime}=\psi(s)+\operatorname{eff}_{N}(\bar{\alpha}) \geq \operatorname{pre}_{N}(\bar{\alpha}+\beta)+\operatorname{eff}_{N}(\bar{\alpha})=\operatorname{pre}_{N}(\alpha+\beta) .
$$

Hence $M^{\prime} \stackrel{\alpha+\beta}{\longrightarrow} N$ and so, by Lemma $5.3(1), s^{\prime} \stackrel{\alpha+\beta}{\longrightarrow} S T S^{\text {rev }}$ and, as a consequence, $s^{\prime} \stackrel{\alpha+\beta}{\longrightarrow}$ STS and $s^{\prime} \stackrel{\beta}{\longrightarrow} S T S$. Hence, by the definition of $S T S^{\text {mrev }}, s^{\prime} \oplus \alpha \stackrel{\bar{\alpha}+\beta}{\longrightarrow} S T S^{\text {mrev }} s^{\prime} \oplus \beta$. Moreover,

$$
\begin{aligned}
& \psi\left(s^{\prime} \oplus \alpha\right)=\psi\left(s^{\prime}\right)+\operatorname{eff}_{N}(\alpha)=M^{\prime}+\operatorname{eff}_{N}(\alpha)=\psi(s)+\operatorname{eff}_{N}(\bar{\alpha})+\operatorname{eff}_{N}(\alpha)=\psi(s) \\
& \psi\left(s^{\prime} \oplus \beta\right)=\psi\left(s^{\prime}\right)+\operatorname{eff}_{N}(\beta)=M^{\prime}+\operatorname{eff}_{N}(\beta)=\psi(s)+\operatorname{eff}_{N}(\bar{\alpha})+\operatorname{eff}_{N}(\beta)=M
\end{aligned}
$$

by Lemma 5.3(3) and Eq.(2).

As the next example shows, reversing a solution of $S T S$ may not lead to a solution of $\overline{S T S}$. Hence, in general, one needs to consider finding solutions to both $S T S$ and $\overline{S T S}$.

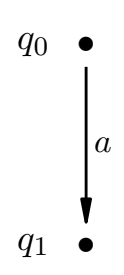

(a)

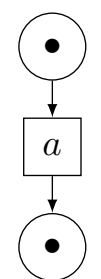

(b)

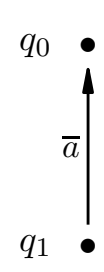

(c)

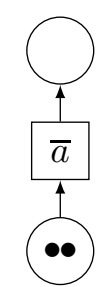

(d)

Figure 5. Reversing a solution does not give a solution to reversing (Example 5.4). 
Example 5.4. Let us consider $S T S$, a step transition system depicted in Figure $5(a)$, and its only home state $q_{1}$. The PT-net $N$ depicted Figure $5(b)$ solves STS. However, the direct reverse of $N$ with the initial marking corresponding to $q_{1}$, depicted in Figure $5(d)$, does not solve the step transition system $\overline{S T S}_{q_{1}}$ shown in Figure 5 (c).

As the set of all the states of a step transition system is a home set, Theorem 5.2 is fundamental as it provides a way of solving mixed reversibility using (much) simpler synthesis problems. In particular, if one is interested whether the mixed reverse $C R G_{N}^{m r e v}$ of the concurrent reachability graph of a PT-net $N$ is solvable when $C R G_{N}$ has a home state.

Theorem 5.5. If $r$ is a home state of $S T S$, then $S T S^{m r e v}$ is solvable if and only if both $S T S$ and $\overline{S T S}_{r}$ are solvable.

\section{Proof:}

Follows directly from Theorems 5.2 .

The above result and the proof of Theorem 5.2 provide a method for constructing a PT-net implementing mixed step reversibility provided that one can synthesise PT-nets for two step transition systems using, e.g., theory of regions [25, 26].

The method for checking the solvability of mixed reversibility easily extends to checking direct reversibility of set transition systems.

Theorem 5.6. Let $S T S$ be a set transition system and $r$ be a home state of $S T S$. Then $S T S^{r e v}$ is solvable if and only if both $S T S$ and $\overline{S T S}_{r}$ are solvable.

\section{Proof:}

$(\Longrightarrow)$ Let $S T S^{r e v} \simeq_{\psi} C R G_{N}$. Then $S T S \simeq_{\psi} C R G_{\left.N\right|_{T}}$ and $\overline{S T S}_{r} \simeq_{\psi} C R G_{N^{\prime}}$, where $N^{\prime}$ is $\left.N\right|_{T}$ with the initial marking set to $\psi(r)$.

$(\Longleftarrow)$ Follows from Theorems 5.2 and 4.4

\section{From sequential reversibility to step reversibility}

Checking the feasibility of step reversibility is, in general, a difficult task. The next result shows that in certain cases it is possible to proceed more effectively, if one is given a PT-net that solves the original step transition system, over-approximates its reverse containing only spikes, and under-approximates its mixed reverse.

Theorem 6.1. Let $S T S=\left(S, T, \rightarrow, s_{0}\right)$ be a CEST-system and $N=\left(P, T \cup \bar{T}, F, M_{0}\right)$ be a PT-net such that:

$$
\left(S T S^{\text {spike }}\right)^{r e v} \triangleleft C R G_{N} \triangleleft S T S^{m r e v} \quad \text { and } \quad S T S \simeq C R G_{\left.N\right|_{T}} .
$$

Then $S T S^{m r e v}$ is solvable. 


\section{Proof:}

The states as well as the initial states of $\left(S T S^{\text {spike }}\right)^{r e v}, S T S^{m r e v}$, and $S T S$ are the same. Moreover, $\left(\left.\left(S T S^{\text {spike }}\right)^{r e v}\right|_{T}\right)^{\text {seq }}=\left(\left.S T S^{m r e v}\right|_{T}\right)^{\text {seq }}=S T S^{\text {seq }}$. Similarly, the initial states of $C R G_{N}$ and $C R G_{\left.N\right|_{T}}$ are the same and we have $\left.\left(C R G_{N}\right)\right|_{T}=C R G_{\left.N\right|_{T}}$. We also observe that all step transition systems in Eq. (3) are CEST-systems, and there is a unique bijection $\psi$ such that:

$$
\left(S T S^{\text {spike }}\right)^{\text {rev }} \triangleleft_{\psi} C R G_{N} \quad C R G_{N} \triangleleft_{\psi^{-1}} S T S^{\text {mrev }} \quad S T S \simeq_{\psi} C R G_{\left.N\right|_{T}} .
$$

By the first part of Eq. (3), SEQ, and the fact that we may assume that each action in $T$ appears in the labels of the transitions of $S T S$, we have:

$$
\operatorname{reach}_{N}=\operatorname{reach}_{\left.N\right|_{T}} \quad \text { and } \quad \operatorname{eff}_{N}(a)=-\operatorname{eff}_{N}(\bar{a}) \text { for every } a \in T .
$$

Lemma 6.2. It can be assumed that $\operatorname{pre}_{N}(\bar{a}) \geq \operatorname{post}_{N}(a)$ and $\operatorname{post}_{N}(\bar{a}) \geq \operatorname{pre}_{N}(a)$, for every $a \in T$.

\section{Proof:}

[Lemma 6.2] Suppose that $F(p, \bar{a})<F(a, p)$, and so also $F(\bar{a}, p)>F(p, a)$. We then modify $F$ to become $F^{\prime}$ which is the same as $F$ except that $F^{\prime}(p, \bar{a})=F(a, p)$ and $F^{\prime}(\bar{a}, p)=F(p, a)$. Let $N^{\prime}$ be the resulting PT-net. Clearly, eff $N_{N}=$ eff $_{N^{\prime}}$.

After this modification, which does not affect actions in $T$, the second part of Eq. (3) is still satisfied after taking $N^{\prime}$ to play the role of $N$. However, the first part of Eq.(3) needs to be demonstrated.

We observe that the modification can only restrict the enabling of steps involving $\bar{a}$. Hence, if the first part of Eq. (3) does not hold with $N^{\prime}$ playing the role of $N$, then there is $M \in \operatorname{reach}_{N^{\prime}} \subseteq \operatorname{reach}_{N}$ and $k \geq 1$ such that $M \stackrel{\bar{a}^{k}}{\longrightarrow} M^{\prime}(*)$ and $\neg M \stackrel{\bar{a}^{k}}{\longrightarrow} N^{\prime}(* *)$. By Eq.(5) and (*), we have $M^{\prime} \stackrel{a^{k}}{\longrightarrow}{ }_{N}$ $M$, and so $M(p) \geq \operatorname{post}_{N}\left(a^{k}\right)(p)(* * *)$.

By construction, (**) implies pre ${ }_{N^{\prime}}\left(\bar{a}^{k}\right)(p)>M(p)$. Thus, by $\operatorname{pre}_{N^{\prime}}\left(\bar{a}^{k}\right)(p)=\operatorname{post}_{N}\left(a^{k}\right)(p)$, we obtain $\operatorname{post}_{N}\left(a^{k}\right)(p)>M(p)$, yielding a contradiction with (***).

We can apply the above modification as many times as needed, finally concluding that the result holds, as any modification does not invalidate the conditions captured in the formulation of this lemma that were obtained by the previous modifications.

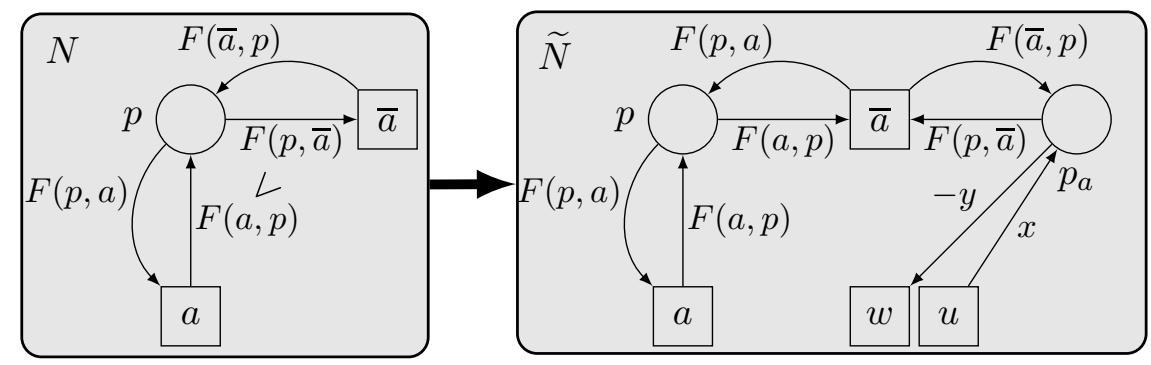

Figure 6. Introducing place $p_{a}$ in the proof of Theorem 6.1 where $u$ represents any place in $T \cup \bar{T} \backslash\{\bar{a}\}$ for which $x=\operatorname{eff}_{N}(u)(p)>0$, and $w$ any place for which $y=\operatorname{eff}_{N}(w)(p) \leq 0$. 
We will show that $S T S^{m r e v}$ is solvable by a PT-net $\widetilde{N}=\left(\widetilde{P}, T \cup \bar{T}, \widetilde{F}, \widetilde{M_{0}}\right)$ constructed thus:

- $\widetilde{P}=\bigcup_{p \in P} P_{p}$, where, for every $p \in P \sqrt{6} P_{p}=\{p\} \cup\left\{p_{a} \mid a \in T \wedge F(p, \bar{a})>F(a, p)\right\}$ and $\widetilde{M}_{0}(q)=M_{0}(p)$ for $q \in P_{p}$.

- The connections in $\widetilde{N}$ are set as follows, where $p \in P$ and $u \in T \cup \bar{T} \backslash\{\bar{a}\}$ :

$$
\begin{aligned}
& \text { - } \widetilde{F}(p, \bar{a})=F(a, p) \text { and } \widetilde{F}(\bar{a}, p)=F(p, a) . \\
& \text { - } \widetilde{F}\left(p_{a}, \bar{a}\right)=F(p, \bar{a}) \text { and } \widetilde{F}\left(\bar{a}, p_{a}\right)=F(\bar{a}, p) . \\
& \text { - } \operatorname{eff}_{N}(u)(p)>0 \text { implies } \widetilde{F}\left(p_{a}, u\right)=0 \text { and } \widetilde{F}\left(u, p_{a}\right)=\operatorname{eff}_{N}(u)(p) . \\
& \text { - } \operatorname{eff}_{N}(u)(p) \leq 0 \text { implies } \widetilde{F}\left(u, p_{a}\right)=0 \text { and } \widetilde{F}\left(p_{a}, u\right)=-\operatorname{eff}_{N}(u)(p) . \\
& \text { - } \widetilde{F} \text { on }(P \times T) \cup(T \times P) \text { is as } F \text { unless it has been set explicitly above. }
\end{aligned}
$$

In what follows, for every marking $M$ of $N$, we use $\phi(M)$ to denote the marking of $\widetilde{N}$ such that $\phi(M)(q)=M(p)$, for every $q \in P_{p}$ and $p \in P$. Hence $\phi\left(M_{0}\right)=\widetilde{M}_{0}$.

We now present a number of straightforward properties of $\tilde{N}$. We first observe that, by Lemma 6.2 for all $a \in T, u \in T \cup \bar{T}$, and $p \in P$,

$$
\begin{aligned}
\operatorname{pre}_{\widetilde{N}}(\bar{a}) \geq \operatorname{post}_{\widetilde{N}}(a) & \operatorname{eff}_{\widetilde{N}}(a)=-\operatorname{eff}_{\widetilde{N}}(\bar{a}) \\
\operatorname{post}_{\widetilde{N}}(\bar{a}) \geq \operatorname{pre}_{\widetilde{N}}(a) & \operatorname{eff}_{\widetilde{N}}(u)\left(P_{p}\right)=\left\{\operatorname{eff}_{N}(u)(p)\right\} .
\end{aligned}
$$

Therefore, for every marking $M$ of $N$ and every $\kappa \in \operatorname{mult}(T \cup \bar{T})$ such that $M+\operatorname{eff}_{N}(\kappa) \geq \varnothing$,

$$
\phi(M)+\operatorname{eff}_{\widetilde{N}}(\kappa)=\phi\left(M+\operatorname{eff}_{N}(\kappa)\right) .
$$

The construction does not affect the enabling of steps involving just one action as well as steps $\alpha$ over $T$ since $p_{a} \in P_{p}$ cannot disable $\alpha$ if it is not also disabled by $p$. Hence, for all markings $M$ of $N$, $u \in T \cup \bar{T}, k \geq 1$, and $\alpha \in \operatorname{mult}(T)$ :

$$
M \stackrel{u^{k}}{\longrightarrow}{ }_{N} \Longleftrightarrow \phi(M) \stackrel{u^{k}}{\longrightarrow} \widetilde{N} \quad \text { and } \quad M \stackrel{\alpha}{\longrightarrow} \stackrel{\alpha}{\longrightarrow}_{\widetilde{N}} .
$$

Thus, by Eqs. (478) and $\widetilde{M}_{0}=\phi\left(M_{0}\right)$,

$$
\left(S T S^{\text {spike }}\right)^{r e v} \triangleleft_{\phi \circ \psi} C R G_{\widetilde{N}} \quad \text { and } \quad S T S \simeq_{\phi \circ \psi} C R G_{\left.\widetilde{N}\right|_{T}} \simeq_{\phi^{-1}} C R G_{\left.N\right|_{T}} .
$$

Lemma 6.3. Let $\alpha, \beta \in \operatorname{mult}(T)$ and $\widetilde{M}=\phi(M)$, for some $M \in \operatorname{mult}(P)$.

1. $\widetilde{M} \stackrel{\bar{\alpha}+\beta}{\longrightarrow} \widetilde{N}$ implies $\widetilde{M}-\operatorname{eff}_{\widetilde{N}}(\alpha) \stackrel{\alpha+\beta}{\longrightarrow} \widetilde{N} \widetilde{M}+\operatorname{eff}_{\widetilde{N}}(\beta)$.

2. $\widetilde{M} \stackrel{\alpha+\beta}{\longrightarrow} \widetilde{N}$ implies $\widetilde{M}+\operatorname{eff}_{\widetilde{N}}(\alpha) \stackrel{\bar{\alpha}+\beta}{\longrightarrow} \widetilde{N} \widetilde{M}+\operatorname{eff}_{\widetilde{N}}(\beta)$.

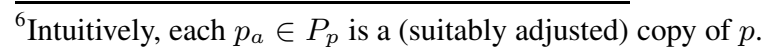




\section{Proof:}

[Lemma 6.3] (1) We first observe that, by $S E Q, \widetilde{M}-\operatorname{eff}_{\widetilde{N}}(\alpha)=\widetilde{M}+\operatorname{eff}_{\widetilde{N}}(\bar{\alpha}) \in \operatorname{reach}_{\widetilde{N}}$. We then observe that, by $\widetilde{M} \geq \operatorname{pre}_{\widetilde{N}}(\bar{\alpha}+\beta)$, the step $\alpha+\beta$ is enabled at $\widetilde{M}-\operatorname{eff}_{\widetilde{N}}(\alpha)$, and so, by Eq.(6):

$\widetilde{M}-\operatorname{eff}_{\widetilde{N}}(\alpha) \geq \operatorname{pre}_{\widetilde{N}}(\bar{\alpha}+\beta)-\operatorname{eff}_{\widetilde{N}}(\alpha)=\operatorname{pre}_{\widetilde{N}}(\bar{\alpha})+\operatorname{pre}_{\widetilde{N}}(\beta)-\operatorname{post}_{\widetilde{N}}(\alpha)+\operatorname{pre}_{\widetilde{N}}(\alpha) \geq \operatorname{pre}_{\widetilde{N}}(\alpha+\beta)$.

Hence, the result holds, as $\widetilde{M}-\operatorname{eff}_{\widetilde{N}}(\alpha)+\operatorname{eff}_{\widetilde{N}}(\alpha+\beta)=\widetilde{M}+\operatorname{eff}_{\widetilde{N}}(\beta)$.

(2) By $S E Q, \widetilde{M} \stackrel{\alpha}{\longrightarrow} \widetilde{N} \widetilde{M}+\operatorname{eff}_{\widetilde{N}}(\alpha)\left(=M^{\prime}\right)$. Suppose that $M^{\prime} \stackrel{\bar{\alpha}+\beta}{\longrightarrow} \widetilde{N}$ does not hold. Then there is $q \in \widetilde{P}$ such that $\operatorname{pre}_{\widetilde{N}}(\bar{\alpha}+\beta)(q)>M^{\prime}(q)(*)$. Moreover, $\widetilde{M} \geq \operatorname{pre}_{\widetilde{N}}(\alpha+\beta)$. Hence, we have:

$\operatorname{pre}_{\widetilde{N}}(\bar{\alpha}+\beta)(q)>\widetilde{M}(q)+\operatorname{eff}_{\widetilde{N}}(\alpha)(q) \geq \operatorname{pre}_{\widetilde{N}}(\alpha+\beta)(q)+\operatorname{eff}_{\widetilde{N}}(\alpha)(q)=\operatorname{pre}_{\widetilde{N}}(\beta)(q)+\operatorname{post}_{\widetilde{N}}(\alpha)(q)$, and so $\operatorname{pre}_{\widetilde{N}}(\bar{\alpha})(q)>\operatorname{post}_{\widetilde{N}}(\alpha)(q)$. Thus there is $a \in \alpha$ and such that $\widetilde{F}(q, \bar{a})>\widetilde{F}(a, q)$ and so, by the definition of $\widetilde{N}, q=p_{a}$, for some $p \in P$. Now, it follows from the construction of $\widetilde{N}$, that there are $\alpha_{0}, \alpha_{1}, \beta_{0}, \beta_{1}$ and $k \geq 1$ such that $\alpha=a^{k}+\alpha_{0}+\alpha_{1}$ and $\beta=\beta_{0}+\beta_{1}$ and $a \notin \alpha_{0}+\alpha_{1}$ and, for $x=\alpha, \beta$, we have:

$$
\begin{aligned}
& \operatorname{post}_{\widetilde{N}}\left(x_{1}\right)\left(p_{a}\right)=\operatorname{pre}_{\widetilde{N}}\left(x_{0}\right)\left(p_{a}\right)=0=\operatorname{pre}_{\widetilde{N}}\left(\overline{x_{1}}\right)\left(p_{a}\right)=\operatorname{post}_{\widetilde{N}}\left(\overline{x_{0}}\right)\left(p_{a}\right) \\
& \operatorname{pre}_{\widetilde{N}}\left(\overline{x_{0}}\right)\left(p_{a}\right)=\operatorname{post}_{\widetilde{N}}\left(x_{0}\right)\left(p_{a}\right) \quad \operatorname{pre}_{\widetilde{N}}\left(x_{1}\right)\left(p_{a}\right)=\operatorname{post}_{\widetilde{N}}\left(\overline{x_{1}}\right)\left(p_{a}\right) .
\end{aligned}
$$

By SEQ, $\widetilde{M} \stackrel{\alpha_{1}+\beta_{1}}{\longrightarrow} \widetilde{N} \widetilde{M}+\operatorname{eff}_{\widetilde{N}}\left(\alpha_{1}+\beta_{1}\right) \stackrel{a^{k}}{\longrightarrow} \widetilde{N} \widetilde{M}+\operatorname{eff}_{\widetilde{N}}\left(\alpha_{1}+\beta_{1}+a^{k}\right)$. Thus, by Eq.(9), $\widetilde{M}+\operatorname{eff}_{\widetilde{N}}\left(\alpha_{1}+\beta_{1}+a^{k}\right) \stackrel{\bar{a}^{k}}{\longrightarrow} \widetilde{N} \widetilde{M}+\operatorname{eff}_{\widetilde{N}}\left(\alpha_{1}+\beta_{1}\right)$, and so we have:

$$
\begin{aligned}
\widetilde{M}\left(p_{a}\right)+\operatorname{eff}_{\widetilde{N}}\left(\alpha_{1}+\beta_{1}+a^{k}\right)\left(p_{a}\right) & =\widetilde{M}\left(p_{a}\right)+\operatorname{eff}_{\widetilde{N}}\left(a^{k}\right)\left(p_{a}\right)+\operatorname{eff}_{\widetilde{N}}\left(\alpha_{1}+\beta_{1}\right)\left(p_{a}\right) \\
& =\widetilde{M}\left(p_{a}\right)+\operatorname{eff}_{\widetilde{N}}\left(a^{k}\right)\left(p_{a}\right)-\operatorname{pre}_{\widetilde{N}}\left(\alpha_{1}+\beta_{1}\right)\left(p_{a}\right) \\
& \geq \operatorname{pre}_{\widetilde{N}}\left(\bar{a}^{k}\right)\left(p_{a}\right) .
\end{aligned}
$$

We therefore have:

$$
\begin{aligned}
M^{\prime}\left(p_{a}\right) & =M\left(p_{a}\right)+\operatorname{eff}_{\widetilde{N}}\left(a^{k}\right)\left(p_{a}\right)-\operatorname{pre}_{\widetilde{N}}\left(\alpha_{1}\right)\left(p_{a}\right)+\operatorname{post}_{\widetilde{N}}\left(\alpha_{0}\right)\left(p_{a}\right) \\
& \geq \operatorname{pre}_{\widetilde{N}}\left(\bar{a}^{k}\right)\left(p_{a}\right)+\operatorname{pre}_{\widetilde{N}}\left(\beta_{1}\right)\left(p_{a}\right)+\operatorname{post}_{\widetilde{N}}\left(\alpha_{0}\right)\left(p_{a}\right) \\
& =\operatorname{pre}_{\widetilde{N}}\left(\bar{a}^{k}\right)\left(p_{a}\right)+\operatorname{pre}_{\widetilde{N}}\left(\beta_{1}\right)\left(p_{a}\right)+\operatorname{pre}_{\widetilde{N}}\left(\bar{\alpha}_{0}\right)\left(p_{a}\right) \\
& =\operatorname{pre}_{\widetilde{N}}(\bar{\alpha})\left(p_{a}\right)+\operatorname{pre}_{\widetilde{N}}(\beta)\left(p_{a}\right) \\
& =\operatorname{pre}_{\widetilde{N}}(\bar{\alpha}+\beta)\left(p_{a}\right)
\end{aligned}
$$

yielding a contradiction with $(*)$. Thus $M^{\prime} \stackrel{\bar{\alpha}+\beta}{\longrightarrow} \widetilde{N}$ holds. Hence we obtain the result as we have $M^{\prime}+\operatorname{eff}_{\widetilde{N}}(\bar{\alpha}+\beta)=\widetilde{M}+\operatorname{eff}_{\widetilde{N}}(\alpha)+\operatorname{eff}_{\widetilde{N}}(\bar{\alpha}+\beta)=\widetilde{M}+\operatorname{eff}_{\widetilde{N}}(\beta)$.

We now conclude that $S T S^{m r e v} \simeq_{\phi \circ \psi} C R G_{\widetilde{N}}$ holds thanks to Eq. (9) and Lemma 6.3.

The last result leads to a simple sufficient condition for the solvability of direct reversibility in the case that proper multisets are not involved. 
Theorem 6.4. Let $S T S$ be a solvable set CEST-system such that $\left(S T S^{s e q}\right)^{r e v}$ is solvable. Then $S T S^{r e v}$ is solvable.

\section{Proof:}

Referring to the notation and proof of Theorem 6.1, we construct a new net $\widetilde{N}^{\prime}$, by adding to $\widetilde{N}$ a fresh set of (mutex) places $P^{\prime}=\left\{p_{a b} \mid a, b \in T\right\}$, where each $p_{a b}$ is such that $\widetilde{M}_{0}\left(p_{a b}\right)=1$ and has four non-zero connections: $\widetilde{F}\left(a, p_{a b}\right)=\widetilde{F}\left(p_{a b}, a\right)=\widetilde{F}\left(\bar{b}, p_{a b}\right)=\widetilde{F}\left(p_{a b}, \bar{b}\right)=1$.

Since all the steps in $S T S$ are sets $P^{\prime}$ ensure that each step enabled at a reachable marking of $\tilde{N}^{\prime}$ is a subset of $T$ or a subset of $\bar{T}$. Moreover, the enabling of such steps is not affected by adding $P^{\prime}$, so we obtain $S T S^{r e v} \simeq C R G_{\widetilde{N}^{\prime}}$ as $S T S^{m r e v} \simeq C R G_{\widetilde{N}}$ holds by Theorem 6.1.

As the next example shows, modifying the original PT-net in Theorem 6.1 is unavoidable.

Example 6.5. Figure 77 $(a)$ depicts a family $N_{n, m}$ of PT-nets which satisfy the assumptions of Theorem 6.1. We have $C R G_{N_{n, m}} \not \subset S T S^{m r e v}$, where $S T S$ is the step reachability graph of the PT-net obtained from $N_{n, m}$ after deleting actions $\bar{a}$ and $\bar{b}$. However, the construction from the proof of Theorem 6.1 yields the PT-net $C R G_{\widetilde{N}_{n, m}}$, shown in Figure 7(b), satisfying $C R G_{\widetilde{N}_{n, m}} \simeq S T S^{m r e v}$.

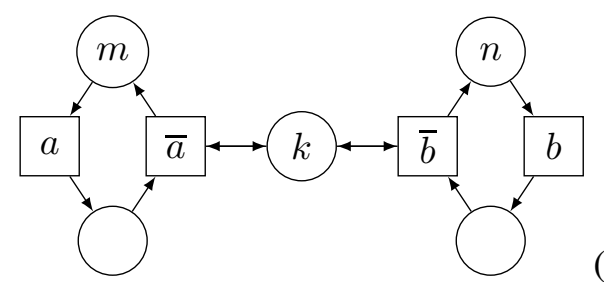

(a)

(b)

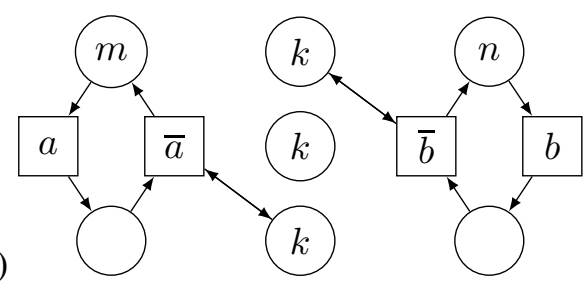

Figure 7. PT-net $N_{n, m}$ with $k=\max (m, n)$ and $m, n \geq 1(a)$; and the same net after applying the construction from Theorem $6.1(b)$.

It is not possible to drop Eq. (3) from the formulation of Theorem 6.1, The next example shows a CEST-system which has only one non-singleton step and is reversible in the sequential semantics, but cannot be reversed in step sequence semantics, even with mixed reverses.

Example 6.6. Let us consider a step transition system $S T S$ together with a PT-net solving it, shown in Figure $8(a, b)$. If we erase the spike between the states $v_{0}$ and $v_{2}$, and add all the reverses (see Figure $8(c)$ ), then the resulting step transition system is solvable (see Figure $8(d)$ ). However, STS cannot be reversed, as shown below.

Suppose that there is a PT-net $N$ solving $S T S^{m r e v}$. Let $M_{i}$ be the marking of $N$ corresponding to the state $v_{i}$, for $i=0, \ldots, 4$. Then the step $(\overline{a a})$ is enabled at $M_{2}$, and $\bar{a}$ is not be enabled at $M_{3}\left(^{*}\right)$.

Let $p$ be any place of $N$. We first observe that $M_{4}$ is a marking, and so $0 \leq M_{4}(p)=M_{2}(p)+2 k$, where $k=\operatorname{eff}_{N}(b)(p)$. Hence $\frac{1}{2} \cdot M_{2}(p)+k \geq 0$. We then recall that $(\overline{a a})$ is enabled at $M_{2}$, and so $M_{2}(p) \geq 2 \cdot F(p, \bar{a})$. Hence $\frac{1}{2} \cdot M_{2}(p) \geq F(p, \bar{a})$. We therefore have:

$$
M_{3}(p)=M_{2}(p)+k=\frac{1}{2} \cdot M_{2}(p)+k+\frac{1}{2} \cdot M_{2}(p) \geq 0+F(p, \bar{a})=F(p, \bar{a}) .
$$

This means that $\bar{a}$ is a step enabled at $M_{3}$, yielding a contradiction with (*). 
(a)

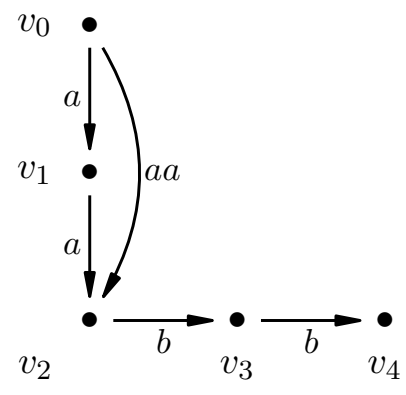

(c)

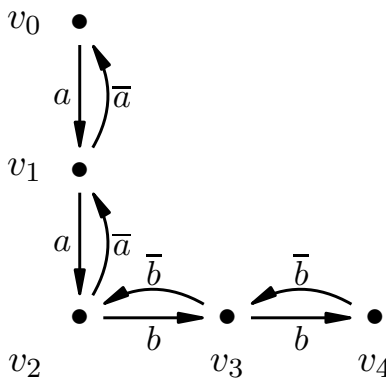

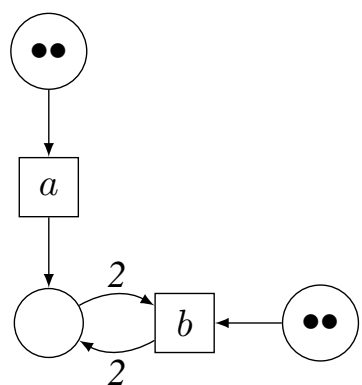

(b)

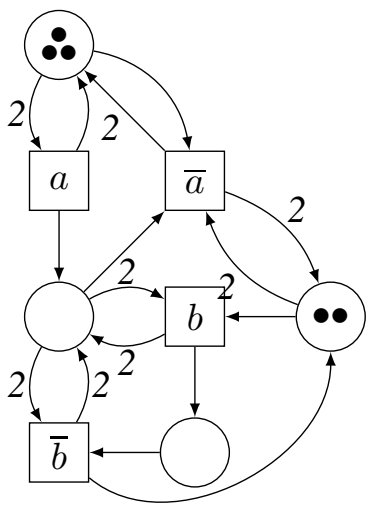

$(d)$

Figure 8. A step transition system $S T S$ with one spike ( $a$ ), and a PT-net solving it (b). $S T S$ without the spike between $v_{0}$ and $v_{2}$ can be reversed $(c, d)$, but $S T S$ cannot.

One might expect that, as it was shown to be the case for bounded PT-nets executed under the sequential semantics [19], it is sufficient to use PT-nets with split reverses also for the reversing under the step semantics. This, however, is not the case as demonstrated in the following example.

Example 6.7. Let us consider a step transition system $S T S$ together with a PT-net solving it, shown in Figure $9(a, b)$. Suppose that there is a PT-net $N$ with split reverses such that $C R G_{N}$ is a split reverse of STS. Moreover, let $M_{i}$ be the marking of $N$ corresponding to $v_{i}$, for $i=1, \ldots, 6$.

Let $p$ be any place of $N$. We first observe that the effect of executing the sequences of actions $a a a$ and $b b$ on $p$ is the same, when going from $M_{1}$ to $M_{6}$. Hence, $3 \cdot \operatorname{eff}_{N}(a)(p)=2 \cdot \operatorname{eff}_{N}(b)(p)$, and so there is an integer $k$ such that eff ${ }_{N}(a)(p)=2 k$ and eff $_{N}(b)(p)=3 k$. With this observation, and by considering different arrows in $S T S$, we obtain:

$$
\begin{array}{lll}
M_{2}(p)=M_{1}(p)+2 k & M_{3}(p)=M_{1}(p)+3 k & M_{4}(p)=M_{1}(p)+5 k \\
M_{5}(p)=M_{1}(p)+4 k & M_{6}(p)=M_{1}(p)+6 k . &
\end{array}
$$

Hence, in particular, we have:

$$
M_{3}(p) \leq M_{5}(p) \leq M_{4}(p) \quad \text { or } \quad M_{3}(p) \geq M_{5}(p) \geq M_{4}(p) .
$$



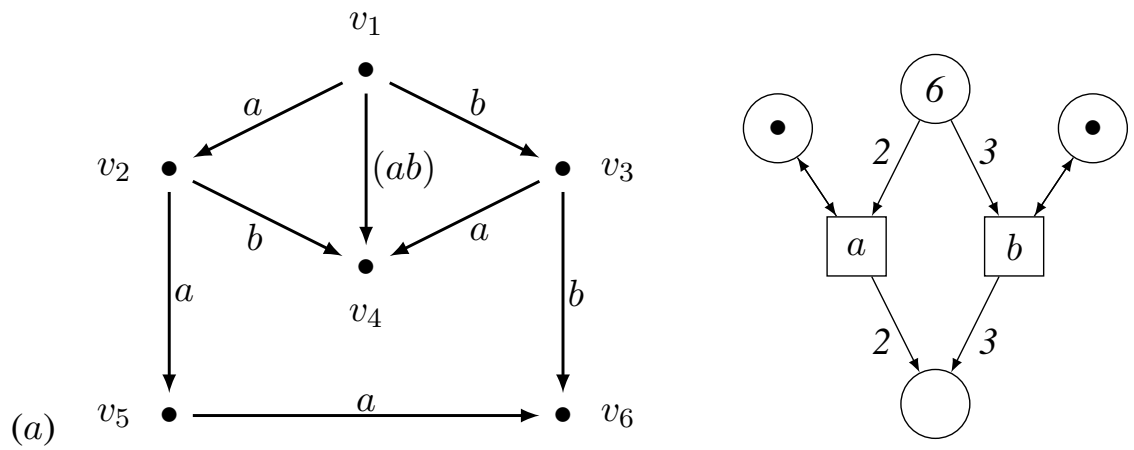

(b)

Figure 9. Splitting is not enough to guarantee reversing (Example 6.7). Note that $v_{1}$ is the initial state.

Suppose now that $\left(\bar{a}_{\langle i\rangle} \bar{b}_{\langle j\rangle}\right)$ is a step reversing $(a b)$ at $M_{4}$. Then, by $S E Q$ and $C E$ holding for the concurrent reachability graphs of PT-nets, $\bar{b}_{\langle j\rangle}$ is also enabled at $M_{3}$. On the other hand, $\bar{b}_{\langle j\rangle}$ is not enabled at $M_{5}$. Then there must be a place $p$ of $N$ such that $M_{5}(p)<\operatorname{pre}_{N}\left(\bar{b}_{\langle j\rangle}\right)(p)$. But we also have $M_{3}(p) \geq \operatorname{pre}_{N}\left(\bar{b}_{\langle j\rangle}\right)(p)$ and $M_{4}(p) \geq \operatorname{pre}_{N}\left(\bar{b}_{\langle j\rangle}\right)(p)$, as $\bar{b}_{\langle j\rangle}$ is enabled at $M_{3}$ and $M_{4}$. This, however, produces a contradiction with Eq.(10).

Example 6.7 can be used further to show that even allowing inhibitor arcs in $N$ would not help 7 The reason is that due to the formulas Eq.(10) for the markings $M_{3}, M_{4}$, and $M_{5}$, no inhibitor place $p$ could be empty at $M_{3}$ and $M_{4}$, and contain a token at $M_{5}$. It would therefore be useless to block $\bar{b}_{\langle j\rangle}$ at $M_{5}$ and still allow the execution of $\bar{b}_{\langle j\rangle}$ at $M_{3}$ and $M_{4}$. Thus, reversing using PT-nets with inhibitor arcs is also not going to work in the general case, when considering the step semantics. This justifies the need to use test arcs 'stronger' than inhibitor arcs in addition to the splitting of reverse actions. Indeed, a general solution can then be obtained using an extended model of PT-nets, as shown in the next section.

\section{A solution combining splitting and weighted read arcs}

A PT-net with weighted read arcs (or PTR-net) is a tuple $N=\left(P, T, F, R, M_{0}\right)$ such that $N^{\prime}=$ $\left(P, T, F, M_{0}\right)$ is a PT-net, and $R: P \times T \rightarrow \mathbb{N}$ is a partial function defining read arcs. All the notations and concepts introduced for $N^{\prime}$ are applicable to $N$ except that a step $\alpha$ of $N$ is enabled at a marking $M$ if it is enabled at marking $M$ in $N^{\prime}$ and, in addition, $R(p, t)=M(p)$, whenever $a \in \alpha$ and $p \in P$ are such that $R(p, a)$ is defined. Read arcs are depicted as arrows with square arrowheads and labelled by their weights.

As the read arcs do not affect markings which result from firing steps of actions, the concurrent reachability graphs of PTR-nets satisfy $C E$. Although SEQ may fail to hold, it is the case that if $\alpha$ is an enabled step, then each step $\beta \leq \alpha$ is also enabled.

We first show that there is a PT-net with weighted read arcs reversing the reachability graph from Example 6.7

\footnotetext{
${ }^{7}$ An inhibitor arc between a place $p$ and action $t$ means that if $t$ is enabled at a marking $M$, then $M(p)=0$.
} 
Example 7.1. Recall the step transition system and the PT-net from Example 6.7 The construction of a solution comes in two phases. In the first phase, splitting is used to reverse all singleton steps. The result, which uses two reverses for $a$ and two reverses for $b$, is shown in Figure 10 $(b)$. Note that although all the singleton steps are indeed reversed, the only non-singleton step $(a b)$ is not. The second phase of the construction adds reverses for $a$ and $b$ which are simultaneously executable at $M_{4}$, as shown in Figure $10(d)$. A solution is then obtained by joining together Figures $9(\mathrm{~b}),[10(b)$ and $10(d)$, by identifying the places with 6 tokens and the places with 0 tokens.

(a)

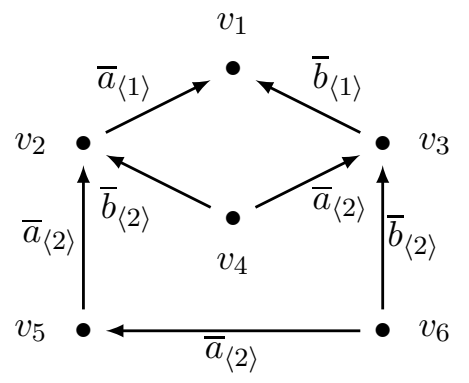

(c)

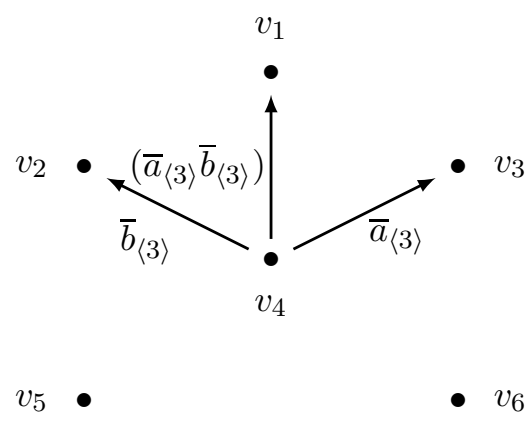

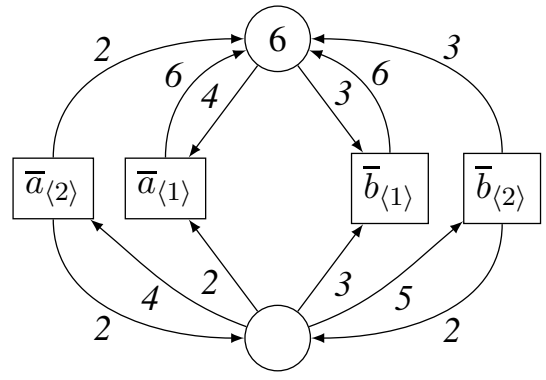

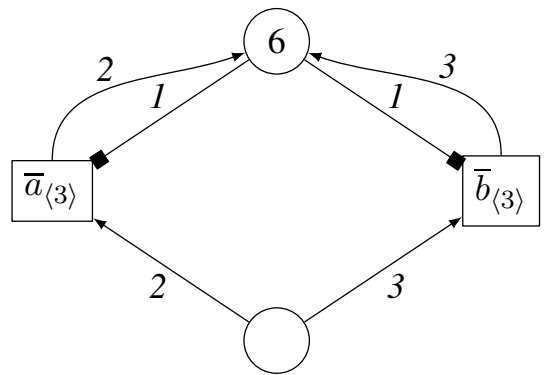

(d)

Figure 10. Reversing with splitting: phase one $(a, b)$, and phase two $(c, d)$.

The solution presented in Example 7.1 inspired the development of a general construction which works for an arbitrary bounded PT-net.

Let $N=\left(P, T, F, M_{0}\right)$ be a bounded PT-net, and let $n$ be an upper limit on the sizes of steps enabled at its reachable markings (such an $n$ always exists as the concurrent reachability graph of $N$ is finite). Moreover, for every marking $M \in \operatorname{reach}_{N}$, the steps annotating actions incoming to $M$ in the concurrent reachability graph $\operatorname{are~in}_{N}(M)=\left\{\alpha \mid \exists M^{\prime} \in \operatorname{reach}_{N}: M^{\prime} \stackrel{\alpha}{\longrightarrow}{ }_{N} M\right\}$. Since $C R G_{N}$ is a CEST-system, $\alpha \leq \beta \in \operatorname{in}_{N}(M)$ implies $\alpha \in \operatorname{in}_{N}(M)$.

We then construct a PTR-net $N^{\prime}=\left(P \uplus P^{\prime}, T \uplus T^{\prime}, F \sqcup F^{\prime}, R, M_{0} \sqcup M_{0}^{\prime}\right)$. A key aspect of the construction is that for each reachable marking $M$ of $N$, and for each maximal step $8 \in \operatorname{in}_{N}(M)$, we add a set of fresh actions $T_{\alpha, M}=\left\{\bar{a}_{\langle\alpha, M, i\rangle} \mid a \in \alpha \wedge 1 \leq i \leq \alpha(a)\right\}$. We then proceed thus:

- For every new action $\bar{a}_{\langle\alpha, M, i\rangle} \in T^{\prime}$ :

$$
-\left.\operatorname{pre}_{N^{\prime}}\left(\bar{a}_{\langle\alpha, M, i\rangle}\right)\right|_{P}=\operatorname{post}_{N}(a) \text { and }\left.\operatorname{post}_{N^{\prime}}\left(\bar{a}_{\langle\alpha, M, i\rangle}\right)\right|_{P}=\operatorname{pre}_{N}(a) .
$$

\footnotetext{
${ }^{8}$ That is, $\alpha \leq \beta \in \operatorname{in}_{N}(M)$ implies $\alpha=\beta$.
} 
- For every $b \in T$, we add a fresh (mutex) place, as in Figure11 $(a)$.

- For every $\bar{b}_{\langle\beta, M, j\rangle} \in T^{\prime}$ with $\alpha \neq \beta$, we add a fresh (mutex) place, as in Figure $11(b)$.

- $P \times T^{\prime}$ is the domain of $R$ and $R\left(p, \bar{a}_{\langle\alpha, M, i\rangle}\right)=M(p)$, for all $p \in P$ and $\bar{a}_{\langle\alpha, M, i\rangle} \in T^{\prime}$.

- $M_{0}^{\prime} \in \operatorname{mult}\left(P^{\prime}\right)$ is the marking of the places in $P^{\prime}$ as indicated in Figure 11

(a)
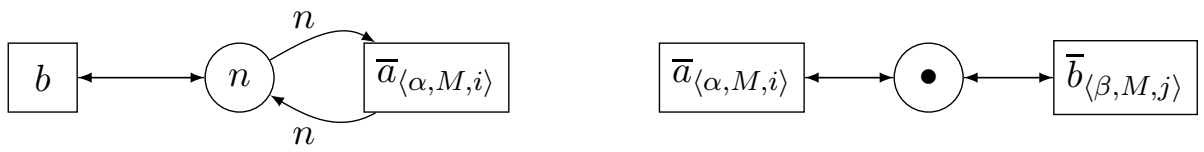

Figure 11. Places $P^{\prime}$ added in the construction of $N^{\prime}$.

We then obtain the desired result.

Theorem 7.2. $C R G_{N^{\prime}}$ is a split reverse of $C R G_{N}$.

\section{Proof:}

Let $S T S=C R G_{N}$ and $S T S^{\prime}=C R G_{N^{\prime}}$. We first gather together some immediate facts about $N^{\prime}$.

\section{Lemma 7.3.}

1. $\bar{a}_{\langle\alpha, M, i\rangle}$ is an indexed reverse of $a$, for all $\bar{a}_{\langle\alpha, M, i\rangle} \in T^{\prime}$ and $a \in T$.

2. $\operatorname{eff}_{N^{\prime}}(\alpha)=\operatorname{eff}_{N}(\alpha) \sqcup \varnothing_{P^{\prime}}$, for every $\alpha \in \operatorname{mult}(T)$.

3. $\operatorname{eff}_{N^{\prime}}(\gamma)=-\operatorname{eff}_{N}(\alpha) \sqcup \varnothing_{P^{\prime}}$, for all $\gamma \in \operatorname{mult}\left(T^{\prime}\right)$ and $\alpha \in \operatorname{mult}(T)$ such that $\bar{\alpha}=\operatorname{noidx}(\gamma)$.

4. $\left.M\right|_{P^{\prime}}=M_{0}^{\prime}$, for every $M \in \operatorname{reach}_{N^{\prime}}$.

5. If $\gamma$ is a step enabled at $M \in \operatorname{reach}_{N^{\prime}}$, then $\gamma \in \operatorname{mult}(T)$, or there is $\alpha \in \operatorname{in}_{N}(M)$ such that $\gamma$ is a set included in $T_{\alpha, M} \subseteq T^{\prime}$.

\section{Proof:}

[Lemma 7.3] (1,2) Follow directly from the definition of $N^{\prime}$.

(3) Follows from part (1).

(4) Follows from parts (2) and (3).

(5) By part (4), $\left.M\right|_{P^{\prime}}=M_{0}^{\prime}$. Hence the result follows from the presence of the weighted read arcs $R$ and the mutex places shown in Figure 11.

We will show that $\operatorname{reach}_{N^{\prime}}=\left\{M \sqcup M_{0}^{\prime} \mid M \in \operatorname{reach}_{N}\right\}$ and $S T S^{r e v} \simeq_{\psi} \operatorname{noidx}\left(S T S^{\prime}\right)$, where $\psi(M)=M \sqcup M_{0}^{\prime}$, for every $M \in \operatorname{reach}_{N}$. 
We first observe that $\psi\left(M_{0}\right)=M_{0} \sqcup M_{0}^{\prime}$ is the initial marking of $N^{\prime}$. Suppose that $M \in \operatorname{reach}_{N}$ is such that $\psi(M)=M \sqcup M_{0}^{\prime} \in$ reach $_{N^{\prime}}$. To show that the executions of steps are preserved by $\psi$ in both directions, we consider four cases, after taking into account Lemma 7.3 (5).

Case 1: $M \stackrel{\alpha}{\longrightarrow}$ STS $M^{\prime}$. Then, since $n$ in Figure 11 $(a)$ is such that $|\alpha| \leq n$, the addition of the new places $P^{\prime}$ does not block $\alpha$. Hence $\alpha$ is enabled at $M \sqcup M_{0}^{\prime}$. Moreover, by Lemma 7.3 (2), $M \sqcup M_{0}^{\prime} \stackrel{\alpha}{\longrightarrow}{ }_{S T S^{\prime}} M^{\prime} \sqcup M_{0}^{\prime}$.

Case 2: $M \stackrel{\bar{\alpha}}{\longrightarrow}{ }_{S T S^{r e v}} M^{\prime}$. Then $M^{\prime} \stackrel{\alpha}{\longrightarrow}_{S T S} M$ and $\alpha \in \operatorname{in}_{N}(M)$. Let $\beta$ be any maximal step in $\operatorname{in}_{N}(M)$ such that $\alpha \leq \beta$ (such a step exists since $C R G_{N}$ is finite). Then there is a subset $\gamma$ of $T_{\beta, M}$ such that noidx $(\gamma)=\bar{\alpha}$. By construction, $\gamma$ is enabled at $M \sqcup M_{0}^{\prime}$. Hence, by Lemma 7.3 (3), $M \sqcup M_{0}^{\prime} \stackrel{\gamma}{\longrightarrow}_{S T S^{\prime}} M^{\prime} \sqcup M_{0}^{\prime}$.

Case 3: $M \sqcup M_{0}^{\prime} \stackrel{\alpha}{\longrightarrow}_{S T S^{\prime}} M^{\prime}$ and $\alpha \in \operatorname{mult}(T)$. Then, by construction and Lemma 7.3 (2), $\alpha$ is enabled at $M$ and $M^{\prime}=\left(M+\operatorname{eff}_{N}(\alpha)\right) \sqcup M_{0}^{\prime}$. Moreover, $M \stackrel{\alpha}{\longrightarrow} S_{S S^{r e v}} M+\operatorname{eff}_{N}(\alpha)$.

Case 4: $M \sqcup M_{0}^{\prime} \stackrel{\gamma}{\longrightarrow}_{S T S^{\prime}} M^{\prime}$, where $\gamma$ is a subset of $T_{\alpha, M}$ for some $\alpha \in \operatorname{in}_{N}(M)$. Let $\beta=$ $\operatorname{noidx}(\gamma) \leq \bar{\alpha}$. Then, by construction and Lemma 7.3 (3), $M^{\prime}=\left(M-\operatorname{eff}_{N}(\beta)\right) \sqcup M_{0}^{\prime}$, $\beta$ is enabled at $M-\operatorname{eff}_{N}(\beta)$, and $M-\operatorname{eff}_{N}(\beta) \stackrel{\beta}{\longrightarrow} S T S M$. Hence $M \stackrel{\bar{\beta}}{\longrightarrow} \operatorname{STS}^{\text {rev }} M-\operatorname{eff}_{N}(\beta)$.

We have developed a general construction which brings us to the same level of reversibility as in the sequential case. However, we had to pay the (costly) price of using of a non-standard class of read arcs. The construction presented above is far from being optimal. Taking as an example the solution from Example 7.1, we observe that it would introduce 5 reverses of $a, 4$ reverses of $b$, and a total of 31 additional places. One can easily see that a large number of them could be avoided, by considering the conditions that force the introduction of each split reversal and those requiring the addition of the new control places. We expect that the proposed construction could be optimised by reducing the number of split reverses and, at the same time, allowing some them to exhibit autoconcurrency (which is admitted in PTR-nets).

\section{Concluding remarks}

In this paper, we continued a study of reversibility in PT-nets, when the step semantics based on executing steps (multisets) of actions rather than single actions is considered, thus capturing real parallelism. In a more abstract setting, the (partial) reversal of steps, thus generating mixed steps possibly containing both original and reverse action, has been studied in [6]. Here we discussed how such reversing can be done in a concrete operational framework of PT-nets.

In the future work, we plan to develop an effective solution to the synthesis problem for the step transition systems with multiple initial states, and address the optimisation of the general solution based on PTR-nets presented in the last section.

Acknowledgement We would like to thank the anonymous reviewers for their careful reading many insightful comments and suggestions for improvement. 
This research was supported by the EU Cost Action IC1405 and the Polish National Agency for Academic Exchange under Grant PPI/APM/2018/1/00036/U/001. The first author was partially supported by projects TIN2015-67522-C3-3-R, PID2019-108528RB-C22, and by Comunidad de Madrid as part of the program S2018/TCS-4339 (BLOQUES-CM) co-funded by EIE Funds of the European Union. The third author was partially supported by the Polish NCN Grant 2017/27/B/ST6/02093.

\section{References}

[1] Danos V, Krivine J. Reversible Communicating Systems. In: Proc. of CONCUR'04, volume 3170 of LNCS, pp. 292-307. 2004. doi:10.1007/978-3-540-28644-8_19.

[2] Danos V, Krivine J. Transactions in RCCS. In: Proc. of CONCUR'05, volume 3653 of LNCS, pp. 398 412. 2005. doi:10.1007/11539452_31.

[3] Cohen M. Systems for financial and electronic commerce, 2013. US Patent 8,527,406.

[4] Phillips I, Ulidowski I. Reversing algebraic process calculi. J. of Log. and Alg. Prog., 2007. 73(1-2):70-96. doi:10.1016/j.jlap.2006.11.002.

[5] Lanese I, Mezzina C, Stefani JB. Reversing Higher-Order Pi. In: Proc. of CONCUR'10, volume 6269 of LNCS. 2010 pp. 478-493. doi:10.1007/978-3-642-15375-4_33.

[6] Phillips I, Ulidowski I. Reversibility and asymmetric conflict in event structures. J. of Log. and Alg. Meth. in Prog., 2015. 84(6):781-805. doi:10.1016/j.jlamp.2015.07.004.

[7] Cardelli L, Laneve C. Reversible structures. In: Proc. of CMSB'11. 2011 pp. 131-140. doi:10.1145/2037509.2037529.

[8] Danos V, Krivine J, Sobocinski P. General Reversibility. Electr. Notes Theor. Comp. Sci., 2007. 175(3):7586. doi:10.1016/j.entcs.2006.07.036.

[9] Vos AD. Reversible Computing - Fundamentals, Quantum Computing, and Applications. 2010. doi:10.1002/9783527633999.

[10] Esparza J, Nielsen M. Decidability issues for Petri nets. BRICS Report Series, 1994. 1(8).

[11] Colange M, Baarir S, Kordon F, Thierry-Mieg Y. Crocodile: a Symbolic/Symbolic tool for the analysis of Symmetric Nets with Bag. In: Proc. of ATPN'11. Springer, 2011 pp. 338-347. doi:10.1007/978-3-64221834-7_20.

[12] Hujsa T, Delosme JM, Kordon AM. On the Reversibility of Live Equal-Conflict Petri Nets. In: Proc. of ATPN'15, volume 9115 of LNCS. 2015 pp. 234-253. doi:10.1007/978-3-319-19488-2_12.

[13] Özkan H, Aybar A. A Reversibility Enforcement Approach for Petri Nets Using Invariants. WSEAS Transactions on Systems, 2008. 7:672-681. ISSN:1109-2777.

[14] Melgratti HC, Mezzina CA, Ulidowski I. Reversing P/T Nets. In: COORDINATION 2019, volume 11533 of Lecture Notes in Computer Science. Springer, 2019 pp. 19-36. doi:10.1007/978-3-030-22397-7_2.

[15] Philippou A, Psara K. Reversible Computation in Petri Nets. In: RC 2018, volume 11106 of Lecture Notes in Computer Science. Springer, 2018 pp. 84-101. arXiv:1804.04607 [cs.LO].

[16] Barylska K, Koutny M, Mikulski Ł, Piątkowski M. Reversible computation vs. reversibility in Petri nets. Sci. Comput. Program., 2018. 151:48-60. doi:10.1016/j.scico.2017.10.008. 
[17] Bouziane Z, Finkel A. Cyclic Petri net reachability sets are semi-linear effectively constructible. In: Proc. of Infinity'97. 1997 pp. 15-24. doi:10.1016/S1571-0661(05)80423-2.

[18] Mikulski Ł, Lanese I. Reversing Unbounded Petri Nets. In: Proc. of ATPN'19, volume 11522 of LNCS. Springer, 2019 pp. 213-233. doi:10.1007/978-3-030-21571-2_13.

[19] Barylska K, Erofeev E, Koutny M, Mikulski Ł, Piątkowski M. Reversing Transitions in Bounded Petri Nets. Fundam. Inform., 2018. 157(4):341-357. doi:10.3233/FI-2018-1631.

[20] Barylska K, Best E, Erofeev E, Mikulski Ł, Piątkowski M. Conditions for Petri Net Solvable Binary Words. ToPNoC, 2016. 11:137-159. doi:10.1007/978-3-662-53401-4_7.

[21] Erofeev E, Barylska K, Mikulski Ł, Piątkowski M. Generating All Minimal Petri Net Unsolvable Binary Words. In: Proc. of PSC'16. 2016 pp. 33-46.

[22] de Frutos-Escrig D, Koutny M, Mikulski Ł. An Efficient Characterization of Petri Net Solvable Binary Words. In: Proc. of ATPN'18. 2018 pp. 207-226. doi:10.1007/978-3-319-91268-4_11.

[23] de Frutos-Escrig D, Koutny M, Mikulski Ł. Reversing Steps in Petri Nets. In: Proc. of ATPN'19, volume 11522 of LNCS. Springer, 2019 pp. 171-191. doi:10.1007/978-3-030-21571-2_11.

[24] Kleijn H, Koutny M. Causality Semantics of Petri Nets with Weighted Inhibitor Arcs. In: Proc. of CONCUR'02, volume 2421 of LNCS. Springer, 2002 pp. 531-546. doi:10.1007/3-540-45694-5_35.

[25] Badouel E, Darondeau P. Theory of Regions. In: LNCS 1491. 1996 pp. 529-586.

[26] Darondeau P, Koutny M, Pietkiewicz-Koutny M, Yakovlev A. Synthesis of Nets with Step Firing Policies. Fundam. Inform., 2009. 94(3-4):275-303. doi:10.3233/FI-2009-132.

[27] Reisig W. Understanding Petri Nets - Modeling Techniques, Analysis Methods, Case Studies. 2013. doi:10.1007/978-3-642-33278-4. 